\title{
SPLINES AND WAVELETS ON GEOPHYSICALLY RELEVANT MANIFOLDS
}

\author{
Isaac Z. Pesenson 1
}

\section{Introduction.}

\section{Manifolds and operators.}

2.1. Compact Riemannian manifolds without boundary.

2.2. Compact homogeneous manifolds.

2.3. Bounded domains with smooth boundaries.

2.4. Radon transform on spheres.

2.5. Hemispherical Radon transform on spheres.

2.6. Radon transform on the group of rotations $S O(3)$.

3. Generalized variational splines on compact Riemannian manifolds 3.1 Generalized interpolating variational splines.

3.2. Approximation by pointwise interpolation and approximation.

3.3. A sampling theorem and a cubature formula.

\section{Bandlimited and localized Parseval frames on homogeneous mani- folds}

5. Applications to the Radon transform on $S^{d}$

5.1. Approximate inversion of the spherical Radon transform using generalized splines.

5.2. A sampling theorem for the spherical Radon transform of bandlimited functions on $S^{d}$.

5.3. Exact formulas for Fourier coefficients of a bandlimited function $f$ on $S^{d}$ from a finite number of samples of $R f$.

6. Applications to the Radon transform on $S O(3)$

6.1. Approximate inversion of the Radon transform on $S O(3)$ using generalized splines.

6.2. A sampling theorem for the Radon transform of bandlimited functions on $S O(3)$.

6.3. Exact formulas for Fourier coefficients of a bandlimited function $f$ on $S O(3)$ from a finite number of samples of $\mathcal{R} f$.

\section{Conclusion.}

\section{References.}

1 Department of Mathematics, Temple University, Philadelphia, PA 19122; pesenson@temple.edu. The author was supported in part by the National Geospatial-Intelligence Agency University Research Initiative (NURI), grant HM1582-08-1-0019. 


\section{INTRODUCTION}

The interpolation problem on the unit two dimensional sphere $S^{2}$, the problem of evaluating the Fourier coefficients of functions on $S^{2}$ and closely related problems about approximation and cubature formulas on $S^{2}$ attracted interest of many mathematicians. Analysis on $S^{2}$ found many applications in seismology, weather prediction, astrophysics, signal analysis, computer vision, computerized tomography, neuroscience, and statistics. In the last two decades, the importance of these and other applications triggered the development of various generalized wavelet bases and frames suitable for the unit spheres $S^{2}$ and $S^{3}$ and the rotation group of $\mathbb{R}^{3}$. Our list of references is very far from being complete $1-11$, 13- 28, 31- 36, [40- [55. More references can be found in monographs [19, 20], 34]. Applications of frames on manifolds to scattering theory, to statistics and cosmology can be found in [3], 26], 37]-39], 56].

Cubature formulas on spheres and interpolation on spheres can be traced back to the classical papers by S. L. Sobolev [59] and I. J. Schoenberg [58]. Substantial and in many ways pioneering work on splines, interpolation and approximation on spheres with many applications to geophysics was done by W. Freeden and his collaborators [16-21. G. Wahba 61-62 initiated spherical splines with the aim to advance statistical analysis on spheres. Important construction of the so-called needlets on $S^{2}$ was done by F.J. Narcowich, P. Petrushev and J. Ward 41.

The goal of the present study is to describe new constructions and applications of splines and bandlimited and localized frames in a space $L_{2}(M)$, where $M$ is a compact Riemannian manifold. Our article is a summary of some results for compact manifolds that were obtained in [5], [6], [24], [46]-[55]. To the best of our knowledge these are the papers which contain the most general results about splines, frames and Shannon sampling on compact Riemannian manifolds along with applications to Radon-type transforms on manifolds.

The following classes of manifolds will be considered: compact manifolds without boundary, compact homogeneous manifolds, bounded domains with smooth boundaries in Euclidean spaces. One can think of a manifold as of a surface in a high dimensional Euclidean space. A homogeneous manifold is a surface with "many" symmetries like the sphere $x_{1}^{2}+\ldots+x_{d}^{2}=1$ in Euclidean space $\mathbb{R}^{d}$. An important example of a bounded domain is a ball $x_{1}^{2}+\ldots+x_{d}^{2} \leq 1$ in $\mathbb{R}^{d}$.

Here is a brief description of the paper. In section 2 we briefly introduce situations which are considered in the paper. We introduce some very basic notions which relate to compact Riemannian manifolds and elliptic differential operators on them, compact homogeneous manifolds and Casimir operators, Radon transform (or Funk transform) on the unit sphere $S^{d}$, hemispherical transform on $S^{d}$, Radon transform on the group of rotations $S O(3)$. More details about the Radon transform on general homogeneous manifolds and in particular on $S O(3)$ can be found in [30] and in [4]-9, 32, 33, [56]. In section 2 we formulate two our imprtant results (see [24 and [51]): a Theorem 2.1] about positive cubature formulas on general compact Riemnnian manifolds and Theorem 2.2 about eigenfunctions of a Casimir operator on a compact homogeneous manifold.

In section 3 we introduce what we call generalized variational interpolating splines on compact Riemannian manifolds [46]-50]. We are motivated by the following problem which is of interest for integral geometry. Let $M, \operatorname{dim} M=d$, be a Riemannian manifold and $M_{\nu}, \nu=1,2, \ldots, N$, is a family of submanifolds 
$\operatorname{dim} M_{\nu}=d_{\nu}, 0 \leq d_{\nu} \leq d$. Given a set of numbers $v_{1}, v_{2}, \ldots, v_{N}$ we would like to find a function for which

$$
\int_{M_{\nu}} f d x=v_{\nu}, \quad \nu=1,2, \ldots, N
$$

Moreover, we are interested in a "least curved" function that satisfies the previous conditions. In other words, we seek a function that satisfies (1.1) and minimizes the functional

$$
u \rightarrow\left\|(1-L)^{t / 2} u\right\|, \quad t \in \mathbb{R},
$$

where $L$ is a differential second order elliptic operator which is self-adjoint in the natural space $L_{2}(M)$. Note that in the case when the submanifold $M_{\nu}$ is a point the integral (1.1) is understood as a value of a function at this point.

Our result is that if $s$ is a solution of such variational problem then the distribution $(1-L)^{t} s$ should satisfy the following distributional pseudo-differential equation on $M$ for any $\psi \in C_{0}^{\infty}(M)$,

$$
\int_{M} \psi(1-L)^{t} s d x=\sum_{\nu=1}^{N} \alpha_{\nu} \int_{M \nu} \psi d x
$$

where coefficients $\alpha_{\nu} \in \mathbb{C}$ depend just on $s$. This equation allows one to obtain the Fourier coefficients of the function $s$ with respect to eigen functions of the operator $L$.

From the very definition our solution $s$ is an "interpolant" in the sense that it has a prescribed set of integrals. Moreover, we show that the function $s$ is not just an interpolant but also an optimal approximation to the set of all functions $f$ in the Sobolev space $H_{t}(M)$ that satisfy (1.1) and

$$
\left\|(1-L)^{t / 2} f\right\| \leq K
$$

for appropriate $K>0$. Namely, we show that $s$ is the center of the convex and bounded set of all functions that satisfy (1.1) and (1.3).

In subsection 3.3 we using splines to formulate our generalization of the Shannon's Sampling Theorem for manifolds and to introduce cubature formulas.

In section 4 we construct bandlimited and localized Parseval frames in $L_{2}(M)$ where $M$ is a homogeneous manifold [24, 51].

Let us remind that a set of vectors $\left\{\theta_{v}\right\}$ in a Hilbert space $H$ is called a Hilbert frame if there exist constants $A, B>0$ such that for all $f \in H$

$$
A\|f\|_{2}^{2} \leq \sum_{v}\left|\left\langle f, \theta_{v}\right\rangle\right|^{2} \leq B\|f\|_{2}^{2} .
$$

The largest $A$ and smallest $B$ are called respectively the lower and the upper frame bounds and the ratio $B / A$ is known as the tightness of the frame. If $A=B$ then $\left\{\theta_{v}\right\}$ is a tight frame, and if $A=B=1$ it is called a Parseval frame. Parseval frames are similar in many respects to orthonormal bases. For example, if all members of a Parseval frame are unit vectors then it is an orthonormal basis.

According to the general theory of Hilbert frames [12, 27] the frame inequality (1.4) implies that there exists a dual frame $\left\{\Theta_{v}\right\}$ (which is not unique in general) for which the following reconstruction formula holds

$$
f=\sum_{v}\left\langle f, \theta_{v}\right\rangle \Theta_{v} .
$$

The important fact is that in the case of a Parseval frame one can take $\Theta_{v}=\theta_{v}$. 
Using Theorems 2.1 and 2.2 we construct a Parseval frame in $L_{2}(M)$ (where $M$ is compact and homogeneous) whose elements are bandlimited and have very strong localization on $M$. Such frame is a substitute for a wavelet basis and it is perfectly suitable to perform multiresolution analysis on compact homogeneous manifolds.

In section 5 we apply these results to Radon (Funk) transform $R$ on $S^{d}$ (see [49]). In this case $M=S^{d}$, every $M_{\nu}, \nu=1, \ldots, N$, is a great subsphere $w_{\nu} \subset S^{d}$ and $L$ is the Laplace-Beltrami operator on $S^{d}$. The transform $R$ is invertible on the set of even functions on $S^{d}$ and it transforms even functions into even functions. The objective is to find approximate preimage of $R f$ where $f$ is sufficiently smooth and even by using only the set of values $\left\{v_{\nu}\right\}_{1}^{N}$ defined in (1.1). We achieve the goal (Theorem 5.1) by constructing an even smooth function $s_{t}(f)$ ( $t$ is a smoothness parameter) which interpolates $f$ in the sense that it has the same set of integrals over subspheres $\left\{w_{\nu}\right\}_{1}^{N}$. Although this interpolant is an optimal approximation to $f$ in the sense explained above we are unable to characterize this approximation quantitatively.

In subsection 5.2 we explore a different approach. This approach seems to be more complicated but it allows to obtain a natural quantitative estimate on the rate of convergence of our approximations. We consider a set of points $\left\{x_{\nu}\right\}_{1}^{N}$ which is dual to the set of subspheres $\left\{w_{\nu}\right\}_{1}^{N}$. Let $\rho>0$ be a separation parameter for the mesh $\left\{x_{\nu}\right\}_{1}^{N}$. The first step now is to use values $\left\{R f\left(x_{\nu}\right)\right\}_{1}^{N}$ to construct a spline $s_{\rho, \tau}(R f)(\tau$ is a smoothness parameter) which interpolates and approximates the Radon transform $R f$. Next, we project $s_{\rho, \tau}(R f)$ on the space of even smooth functions to obtain even function $\widehat{s}_{\rho, \tau}(R f)$ which is another (generally a better) approximation to $R f$. Now for the even function $S_{\rho, \tau}(f)=R^{-1} \widehat{s}_{\rho, \tau}(R f)$ we are able to show (see Theorem 5.2 ) that a difference between $S_{\rho, \tau}(f)$ and $f$ is of order $\rho$ to a power. In other words when $\rho$ goes to zero a sequence of corresponding functions $S_{\rho, \tau}(f)$ converges to $f$ with a geometrical rate. Moreover, the Theorem 5.3 shows that if $f$ is $\omega$-bandlimited and $\rho$ is small enough compare to $\omega$ than $S_{\rho, \tau}(f)$ converges to $f$ when smoothness $\tau$ goes to infinity ( for a sufficiently dense but fixed mesh $\left\{x_{\nu}\right\}_{1}^{N}$ ). This result can be considered as a generalization of the Classical Sampling Theorem.

In subsection 5.3 we are using Theorems 2.1 and 2.2 to obtain exact formulas for Fourier coefficients of a bandlimited function $f$ on $S^{d}$ from a finite number of samples of $R f$.

We note that analogous results can be obtained for the hemispherical transform on $S^{d}$ and for bounded domains in $\mathbb{R}^{n}$ with smooth boundaries ([49], [53]).

In section 6 results which are similar to the results of section 5 obtained for the group Radon transform on $S O(3)$ (see [5], 6]).

\section{MANiFOLDS AND OPERATORS}

We describe all situations which will be discussed in the paper.

2.1. Compact Riemannian manifolds without boundary. Let $M$ be a compact Riemannian manifold without boundary and $L$ be a differential second order elliptic operator which is self-adjoint and negatively semi-definite in the space $L_{2}(M)$ constructed using a Riemannian density $d x$. The best known example of such operator is the Laplace-Beltrami which is given in a local coordinate system 
by the formula

$$
\Delta f=\sum_{m, k} \frac{1}{\sqrt{\operatorname{det}\left(g_{i j}\right)}} \partial_{m}\left(\sqrt{\operatorname{det}\left(g_{i j}\right)} g^{m k} \partial_{k} f\right)
$$

where $g_{i j}$ are components of the metric tensor, $\operatorname{det}\left(g_{i j}\right)$ is the determinant of the matrix $\left(g_{i j}\right), g^{m k}$ components of the matrix inverse to $\left(g_{i j}\right)$.

In order to have an invertible operator we will work with $I-L$, where $I$ is the identity operator in $L_{2}(M)$. It is known that for every such operator $L$ the domain of the power $(I-L)^{t / 2}, t \in \mathbb{R}$, is the Sobolev space $H_{t}(M), t \in \mathbb{R}$. There are different ways to introduce norm in Sobolev spaces. For a fixed operator $L$ we will introduce the graph norm as follows.

Definition 2.1. The Sobolev space $H_{t}(M), t \in \mathbb{R}$ can be introduced as the domain of the operator $(I-L)^{t / 2}$ with the graph norm

$$
\|f\|_{t}=\left\|(I-L)^{t / 2} f\right\|, f \in H_{t}(M) .
$$

Note, that such norm depends on $L$. However, for every two differential of order two elliptic operators such norms are equivalent for each $t \in \mathbb{R}$.

Since the operator $(-L)$ is self-adjoint and positive semi-definite it has a discrete spectrum $0 \leq \lambda_{0} \leq \lambda_{1} \leq \lambda_{2} \leq \ldots$, and one can choose corresponding eigenfunctions $u_{0}, u_{1}, \ldots$ which form an orthonormal basis of $L_{2}(M)$. A distribution $f$ belongs to $H_{t}(M), t \in \mathbb{R}$, if and only if

$$
\|f\|_{t}=\left(\sum_{j=0}^{\infty}\left(1+\lambda_{j}\right)^{t}\left|c_{j}(f)\right|^{2}\right)^{1 / 2}<\infty,
$$

where Fourier coefficients $c_{j}(f)$ of $f$ are given by

$$
c_{j}(f)=\left\langle f, u_{j}\right\rangle=\int_{M} f \overline{u_{j}} .
$$

Definition 2.2. The span of all eigenfunctions of $-L$ whose corresponding eigenvalues are not greater than a positive $\omega$ is denoted by $\mathbf{E}_{\omega}(L)$ and called the set of $\omega$-bandlimited functions.

Definition 2.3. For a sufficiently small $\rho>0$ we will say that a finite set of points $M_{\rho}=\left\{x_{\nu}\right\}_{\nu=1}^{N}$ is a $\rho$-lattice, if

(1) The balls $B\left(x_{\nu}, \rho / 2\right)$ are disjoint.

(2) The balls $B\left(x_{\nu}, \rho\right)$ form a cover of $M$.

The following theorem holds for any compact manifold [24, 51].

Theorem 2.1. (Cubature formula with positive weights) There exists a positive constant $c=c(M)$, such that if $\rho=c \omega^{-1 / 2}$, then for any $\rho$-lattice $M_{\rho}=\left\{x_{\nu}\right\}_{\nu=1}^{N}$, there exist strictly positive coefficients $\mu_{\nu}>0$ for which the following equality holds for all functions in $\mathbf{E}_{\omega}(M)$ :

$$
\int_{M} f d x=\sum_{x_{\nu} \in M_{\rho}} \mu_{\nu} f\left(x_{\nu}\right)
$$

Moreover, there exists constants $c_{1}, c_{2}$, such that the following inequalities hold:

$$
c_{1} \omega^{-d / 2} \leq \mu_{\nu} \leq c_{2} \omega^{-d / 2}, d=\operatorname{dim} M .
$$


2.2. Compact homogeneous manifolds. The most complete results will be obtained for compact homogeneous manifolds.

A homogeneous compact manifold $M$ is a $C^{\infty}$-compact manifold on which a compact Lie group $G$ acts transitively. In this case $M$ is necessary of the form $G / K$, where $K$ is a closed subgroup of $G$. The notation $L_{2}(M)$, is used for the usual Hilbert spaces, with invariant measure $d x$ on $M$.

The Lie algebra $\mathbf{g}$ of a compact Lie group $G$ is then a direct sum $\mathbf{g}=\mathbf{a}+[\mathbf{g}, \mathbf{g}]$, where $\mathbf{a}$ is the center of $\mathbf{g}$, and $[\mathbf{g}, \mathbf{g}]$ is a semi-simple algebra. Let $Q$ be a positivedefinite quadratic form on $\mathbf{g}$ which, on $[\mathbf{g}, \mathbf{g}]$, is opposite to the Killing form. Let $X_{1}, \ldots, X_{n}$ be a basis of $\mathbf{g}$, which is orthonormal with respect to $Q$. Since the form $Q$ is $A d(G)$-invariant, the operator

$$
-X_{1}^{2}-X_{2}^{2}-\ldots-X_{n}^{2}, n=\operatorname{dim} G
$$

is a bi-invariant operator on $G$, which is known as the Casimir operator. This implies in particular that the corresponding operator on $L_{2}(M)$,

$$
\mathcal{L}=-D_{1}^{2}-D_{2}^{2}-\ldots-D_{n}^{2}, \quad D_{j}=D_{X_{j}}, n=\operatorname{dim} G,
$$

commutes with all operators $D_{j}=D_{X_{j}}$. The operator $\mathcal{L}$, which is usually called the Laplace operator, is the image of the Casimir operator under differential of quaziregular representation in $L_{2}(M)$. It is important to realize that in general, the operator $\mathcal{L}$ is not necessarily the Laplace-Beltrami operator of the natural invariant metric on $M$. But it coincides with such operator at least in the following cases: 1) If $M$ is a $d$-dimensional torus, 2) If the manifold $M$ is itself a compact semi-simple Lie group group $G$ ([29, Ch. II), 3 ) If $M=G / K$ is a compact symmetric space of rank one ([29], Ch. II, Theorem 4.11).

The following important result was obtained in 24], [51].

Theorem 2.2. (Product property) If $M=G / H$ is a compact homogeneous manifold and $L$ is the same as above, then for any $f$ and $g$ belonging to $\mathbf{E}_{\omega}(\mathcal{L})$, their product $f g$ belongs to $\mathbf{E}_{4 m \omega}(\mathcal{L})$, where $m$ is the dimension of the group $G$.

In the case when $M$ is the rank one compact symmetric space (in particular, the sphere $S^{d}$ or any of the projective spaces $P^{d}, \mathbb{C} P^{d}, \mathbb{Q} P^{d}$ ) there is a better result.

Theorem 2.3. If $M=G / H$ is a compact symmetric space of rank one then for any $f$ and $g$ belonging to $\mathbf{E}_{\omega}(\mathcal{L})$, their product $f g$ belongs to $\mathbf{E}_{2 \omega}(\mathcal{L})$.

2.3. Bounded domains with smooth boundaries. Our consideration includes also an open bounded domain $M \subset \mathbb{R}^{d}$ with a smooth boundary $\Gamma$ which is a smooth $(d-1)$-dimensional oriented manifold. Let $\bar{M}=M \cup \Gamma$ and $L_{2}(M)$ be the space of functions square-integrable with respect to the Lebesgue measure $d x=d x_{1} \ldots d x_{d}$ with the norm denoted as $\|\cdot\|$. If $k$ is a natural number the notations $H^{k}(M)$ will be used for the Sobolev space of distributions on $M$ with the norm

$$
\|f\|_{H^{k}(M)}=\left(\|f\|^{2}+\sum_{1 \leq|\alpha| \leq k}\left\|\partial^{|\alpha|} f\right\|^{2}\right)^{1 / 2}
$$

where $\alpha=\left(\alpha_{1}, \ldots, \alpha_{d}\right)$ and $\partial^{|\alpha|}$ is a mixed partial derivative

$$
\left(\frac{\partial}{\partial x_{1}}\right)^{\alpha_{1}} \ldots\left(\frac{\partial}{\partial x_{d}}\right)^{\alpha_{d}}
$$


Under our assumptions the space $C_{0}^{\infty}(\bar{M})$ of infinitely smooth functions with support in $\bar{M}$ is dense in $H^{k}(M)$. Closure in $H^{k}(M)$ of the space $C_{0}^{\infty}(M)$ of smooth functions with support in $M$ will be denoted as $H_{0}^{k}(M)$.

Since $\Gamma$ can be treated as a smooth Riemannian manifold one can introduce Sobolev scale of spaces $H^{s}(\Gamma), s \in \mathbb{R}$, as, for example, the domains of the LaplaceBeltrami operator $L$ of a Riemannian metric on $\Gamma$.

According to the trace theorem there exists a well defined continuous surjective trace operator

$$
\gamma: H^{s}(M) \rightarrow H^{s-1 / 2}(\Gamma), s>1 / 2,
$$

such that for all functions $f$ in $H^{s}(M)$ which are smooth up to the boundary the value $\gamma f$ is simply a restriction of $f$ to $\Gamma$. One considers the following operator

$$
P f=\sum_{j, k} \partial_{j}\left(a_{j, k}(x) \partial_{k} f\right)
$$

with coefficients in $C^{\infty}(M)$ where the matrix $\left(a_{j, k}(x)\right)$ is real, symmetric and negatively definite on $\bar{M}$. The operator $L$ is defined as the Friedrichs extension of $P$, initially defined on $C_{0}^{\infty}(M)$, to the set of all functions $f$ in $H^{2}(M)$ with constraint $\gamma f=0$. The Green formula implies that this operator is self-adjoint. It is also a positive operator and the domain of its positive square root $(-L)^{1 / 2}$ is the set of all functions $f$ in $H^{1}(M)$ for which $\gamma f=0$. Thus, one has a self-adjoint positive definite operator $(-L)$ in the Hilbert space $L_{2}(M)$ with a discrete spectrum $0<\lambda_{1} \leq \lambda_{2}, \ldots$ which goes to infinity.

One of the most important examples of such domain and operator is the unit ball in $\mathbb{R}^{d}$ with the regular Laplace operator on it. In this case the eigenvalues are $j_{d+\frac{k-2}{2}, l}^{2}$, where $j_{\nu, l}$ is $l$-th positive root of the Bessel function $J_{\nu}$ of first kind and of order $\nu$. The set of eigenfunctions given in spherical coordinates by the formulas

$$
\varphi_{d, i, l}=c_{d, i, l} \rho^{-\frac{k-2}{2}} J_{d+\frac{k-2}{2}}\left(j_{d+\frac{k-2}{2}, l} \rho\right) Y_{d, k}^{i}(\vartheta),
$$

where $k=0,1, \ldots, 1 \leq i \leq n_{d}(k), l=1,2, \ldots$. Constants $c_{d, i, l}$ can be chosen in a way that makes functions $\varphi_{d, i, l}$ normal.

2.4. Radon transform on spheres. We consider the unit sphere $S^{d} \subset \mathbb{R}^{d+1}$ and the corresponding space $L_{2}\left(S^{d}\right)$ constructed with respect to normalized and rotation-invariant measure.

Let $Y_{k}^{i}$ be an orthonormal basis of spherical harmonics in the space $L_{2}\left(S^{d}\right)$, where $k=0,1, \ldots ; i=1,2, \ldots, n_{d}(k)$ and

$$
n_{d}(k)=(d+2 k-1) \frac{(d+k-2) !}{k !(d-1) !}
$$

is the dimension of the subspace of spherical harmonics of degree $k$. Note, that

$$
Y_{k}^{i}(-x)=(-1)^{k} Y_{k}^{i}(x) .
$$

The Fourier decomposition of $f \in L_{2}\left(S^{d}\right)$ is

$$
f=\sum_{i, k} c_{i, k}(f) Y_{k}^{i}
$$

where

$$
c_{i, k}(f)=\int_{S^{d}} f \overline{Y_{k}^{i}} d x=\left\langle f, Y_{k}^{i}\right\rangle_{L_{2}\left(S^{d}\right)} .
$$


To every function $f \in L_{2}\left(S^{d}\right)$ the spherical Radon transform associates its integrals over great subspheres:

$$
R f\left(\theta^{\perp} \cap S^{d}\right)=\int_{\theta^{\perp} \cap S^{d}} f d x
$$

where $\theta^{\perp} \cap S^{d}$ is the great subsphere of $S^{d}$ whose plane has normal $\theta$.

If a function $f \in L_{2}\left(S^{d}\right)$ has Fourier coefficients $c_{i, k}(f)$ then its Radon Transform is given by the formula

$$
R(f)=\pi^{-1 / 2} \Gamma((d+1) / 2) \sum_{i, k} r_{k} c_{i, k}(f) Y_{k}^{i} .
$$

where $Y_{k}^{i}$ are the spherical harmonic polynomials and

$$
\left.r_{k}=(-1)^{k / 2} \Gamma((k+1) / 2)\right) / \Gamma((k+d) / 2)
$$

if $k$ is even and $r_{k}=0$ if $k$ is odd. It implies in particular that operators $\Delta$ and $R$ commute on a set of smooth functions. A function $f \in L_{2}\left(S^{d}\right)$ is said to be even if its Fourier series (2.8) contains only harmonics of even degrees $k=2 m$. Because the coefficients $r_{k}$ have asymptotics $(-1)^{k / 2}(k / 2)^{(1-d) / 2}$ as $k$ goes to infinity we have the following result.

Theorem 2.4. The spherical Radon transform $R$ is a continuous operator from the Sobolev space of even functions $H_{t}^{\text {even }}\left(S^{d}\right)$ onto the space $H_{t+(d-1) / 2}^{\text {even }}\left(S^{d}\right)$. Its inverse $R^{-1}$ is a continuous operator from the space $H_{t+(d-1) / 2}^{\text {even }}\left(S^{d}\right)$ onto the space $H_{t}^{e v e n}\left(S^{d}\right)$. If $f \in H_{t+(d-1) / 2}^{e v e n}\left(S^{d}\right)$ and it has Fourier series $f=\sum_{i, m} c_{i, 2 m}(f) Y_{2 m}^{i}$ then

$$
R^{-1} f=\frac{\sqrt{\pi}}{\Gamma((d+1) / 2)} \sum_{i, m} \frac{c_{i, 2 m}(f)}{r_{2 m}} Y_{2 m}^{i}
$$

2.5. Hemispherical Radon transform on $S^{d}$. To every function $f \in L_{2}\left(S^{d}\right)$ the hemispherical transform $T$ assigns a function $T f \in L_{2}\left(S^{d}\right)$ on the dual sphere $S^{d}$ which is given by the formula

$$
(T f)(\xi)=\int_{\xi \cdot x>0} f(x) d x .
$$

For every function $f \in L_{2}\left(S^{d}\right)$ that has Fourier coefficients $c_{i, j}(f)$ the hemispherical transform can be given explicitly by the formula

$$
T f=\pi^{(d-1) / 2} \sum_{i, k} m_{k} c_{k}^{i}(f) Y_{k}^{i}
$$

where $m_{k}=0$, if $k$ is even and

$$
m_{k}=(-1)^{(k-1) / 2} \frac{\Gamma(k / 2)}{\Gamma((k+d+1) / 2))},
$$

if $k$ is odd.

The transformation $T$ is one to one on the subspace of odd functions (i.e. $f(x)=-f(-x))$ of a Sobolev space $H_{t}^{o d d}\left(S^{d}\right)$ and maps it continuously onto $H_{t+(d+1) / 2}^{o d d}\left(S^{d}\right)$,

$$
T\left(H_{t}^{\text {odd }}\right)\left(S^{d}\right)=H_{t+(d+1) / 2}^{\text {odd }}\left(S^{d}\right) .
$$


2.6. Radon transform on the group of rotations $S O(3)$. The group of rotations $S O(3)$ of $\mathbb{R}^{3}$ consists of $3 \times 3$ real matrices $U$ such that $U^{T} U=I, \operatorname{det} U=1$. It is known that any $g \in S O(3)$ has a unique representation of the form

$$
g=Z(\gamma) X(\beta) Z(\alpha), 0 \leq \beta \leq \pi, 0 \leq \alpha, \gamma<2 \pi,
$$

where

$$
Z(\theta)=\left(\begin{array}{ccc}
\cos \theta & -\sin \theta & 0 \\
\sin \theta & \cos \theta & 0 \\
0 & 0 & 1
\end{array}\right), \quad \mathrm{X}(\theta)=\left(\begin{array}{ccc}
1 & 0 & 0 \\
0 & \cos \theta & -\sin \theta \\
0 & \sin \theta & \cos \theta
\end{array}\right)
$$

are rotations about the $Z$ - and $X$-axes, respectively. In the coordinates $\alpha, \beta, \gamma$, which are known as Euler angles, the Haar measure of the group $S O(3)$ is given as

$$
d g=\frac{1}{8 \pi^{2}} \sin \beta d \alpha d \beta d \gamma
$$

In other words the following formula holds:

$$
\int_{S O(3)} f(g) d g=\int_{0}^{2 \pi} \int_{0}^{\pi} \int_{0}^{2 \pi} f(g(\alpha, \beta, \gamma)) \frac{1}{8 \pi^{2}} \sin \beta d \alpha d \beta d \gamma
$$

Note that if $S O(2)$ is the group of rotations of $\mathbb{R}^{2}$ then the two-dimensional sphere $S^{2}$ can be identified with the factor $S O(3) / S O(2)$.

We introduce Radon transform $\mathcal{R} f$ of a smooth function $f$ defined on $S O(3)$.

Definition 2.4. If $S^{2}$ is the standard unit sphere in $\mathbb{R}^{3}$, then for a pair $(x, y) \in$ $S^{2} \times S^{2}$ the value of the Radon transform $\mathcal{R} f$ at $(x, y)$ is defined by the formula

$$
\begin{gathered}
(\mathcal{R} f)(x, y)=\frac{1}{2 \pi} \int_{\{g \in S O(3): x=g y\}} f(g) d \nu_{g}= \\
4 \pi \int_{S O(3)} f(g) \delta_{y}\left(g^{-1} x\right) d g=\left(f * \delta_{y}\right)(x), \quad(x, y) \in S^{2} \times S^{2},
\end{gathered}
$$

where $d \nu_{g}=8 \pi^{2} d g$, and $\delta_{y}$ is the measure concentrated on the set of all $g \in S O(3)$ such that $x=g y$.

An orthonormal system in $L_{2}\left(S^{2}\right)$ is provided by the spherical harmonics $\left\{Y_{k}^{i}, k \in\right.$ $\left.\mathbb{N}_{0}, i=1, \ldots, 2 k+1\right\}$. The subspaces $\mathcal{H}_{k}:=\operatorname{span}\left\{Y_{k}^{i}, i=1, \ldots, 2 k+1\right\}$ spanned by the spherical harmonics of degree $k$ are the invariant subspaces of the quasiregular representation $T(g): f(x) \mapsto f\left(g^{-1} \cdot x\right)$, (where $\cdot$ denotes the canonical action of $S O(3)$ on $\left.S^{2}\right)$. Representation $T$ decomposes into $(2 k+1)$-dimensional irreducible representation $\mathcal{T}_{k}$ in $\mathcal{H}_{k}$. The corresponding matrix coefficients are the Wigner-polynomials

$$
\mathcal{T}_{k}^{i j}(g)=\left\langle\mathcal{T}_{k}(g) Y_{k}^{i}, Y_{k}^{j}\right\rangle
$$

If $\Delta_{S O(3)}$ and $\Delta_{S^{2}}$ are Laplace-Beltrami operators of invariant metrics on $S O(3)$ and $S^{2}$ respectively, then

$$
\Delta_{S O(3)} \mathcal{T}_{k}^{i j}=-k(k+1) \mathcal{T}_{k}^{i j} \quad \text { and } \quad \Delta_{S^{2}} Y_{k}^{i}=-k(k+1) Y_{k}^{i} .
$$

Using the fact that $\Delta_{S O(3)}$ is equal to $-k(k+1)$ on the eigenspace $\mathcal{H}_{k}$ we obtain

$$
\begin{gathered}
\|f\|_{L_{2}(S O(3))}^{2}=\sum_{k=1}^{\infty}(2 k+1)\left\|(4 \pi)^{-1} \hat{f}(k)\right\|_{L_{2}\left(S^{2} \times S^{2}\right)}^{2}= \\
\left\|(4 \pi)^{-1}\left(I-2 \Delta_{S^{2} \times S^{2}}\right)^{1 / 4} \mathcal{R} f\right\|_{L_{2}\left(S^{2} \times S^{2}\right)}^{2},
\end{gathered}
$$


where $\Delta_{S^{2} \times S^{2}}=\Delta_{1}+\Delta_{2}$ is the Laplace-Beltrami operator of the natural metric on $S^{2} \times S^{2}$. We define the following norm on the space $C^{\infty}\left(S^{2} \times S^{2}\right)$

$$
\|u\| \|^{2}=\left(\left(I-2 \Delta_{S^{2} \times S^{2}}\right)^{1 / 2} u, u\right)_{L_{2}\left(S^{2} \times S^{2}\right)} .
$$

Because $\mathcal{R}$ is essentially an isometry between $L_{2}(S O(3))$ with the natural norm and $L_{2}\left(S^{2} \times S^{2}\right)$ with the norm ||| || $\mid$ the inverse of $\mathcal{R}$ is given by its adjoint operator. To calculate the adjoint operator we express the Radon transform $\mathcal{R}$ in another way. Going back to our problem in crystallography we first state that the great circle $C_{x, y}=\{g \in S O(3): g \cdot x=y\}$ in $S O(3)$ can also be described by the following formula

$$
C_{x, y}=x^{\prime} S O(2)\left(y^{\prime}\right)^{-1}:=\left\{x^{\prime} h\left(y^{\prime}\right)^{-1}, h \in S O(2)\right\}, \quad x^{\prime}, y^{\prime} \in S O(3),
$$

where $x^{\prime} \cdot x_{0}=x, y^{\prime} \cdot x_{0}=y$ and $S O(2)$ is the stabilizer of $x_{0} \in S^{2}$. Hence,

$$
\begin{array}{r}
\mathcal{R} f(x, y)=\int_{S O(2)} f\left(x^{\prime} h\left(y^{\prime}\right)^{-1}\right) d h=4 \pi \int_{C_{x, y}} f(g) d g \\
=4 \pi \int_{S O(3)} f(g) \delta_{y}\left(g^{-1} \cdot x\right) d g, \quad f \in L_{2}(S O(3)) .
\end{array}
$$

By using this representation one can find that the $L^{2}$-adjoint operator of $\mathcal{R}$ is given by

$$
\mathcal{R}^{*} u=(4 \pi) \int_{S^{2}}\left(I-2 \Delta_{S^{2} \times S^{2}}\right)^{1 / 2} u(g \cdot y, y) d y .
$$

Definition 2.5 (Sobolev spaces on $\left.S^{2} \times S^{2}\right)$. The Sobolev space $H_{t}\left(S^{2} \times S^{2}\right), t \in \mathbb{R}$, is defined as the domain of the operator $\left(I-2 \Delta_{S^{2} \times S^{2}}\right)^{\frac{t}{2}}$ with graph norm

$$
\|f\|_{t}=\left\|\left(I-2 \Delta_{S^{2} \times S^{2}}\right)^{\frac{t}{2}} f\right\|_{L^{2}\left(S^{2} \times S^{2}\right)},
$$

and the Sobolev space $H_{t}^{\Delta}\left(S^{2} \times S^{2}\right), t \in \mathbb{R}$, is defined as the subspace of all functions $f \in H_{t}\left(S^{2} \times S^{2}\right)$ such $\Delta_{1} f=\Delta_{2} f$.

Definition 2.6 (Sobolev spaces on $S O(3)$ ). The Sobolev space $H_{t}(S O(3)), t \in \mathbb{R}$, is defined as the domain of the operator $\left(I-4 \Delta_{S O(3)}\right)^{\frac{t}{2}}$ with graph norm

$$
\||f|\|_{t}=\left\|\left(I-4 \Delta_{S O(3)}\right)^{\frac{t}{2}} f\right\|_{L^{2}(S O(3))}, \quad f \in L_{2}(S O(3)) .
$$

It is not difficult to prove the following theorems.

Theorem 2.5. For any $t \geq 0$ the Radon transform on $S O(3)$ is an invertible mapping

$$
\mathcal{R}: H_{t}(S O(3)) \rightarrow H_{t+\frac{1}{2}}^{\Delta}\left(S^{2} \times S^{2}\right)
$$

and

$$
f(g)=\int_{S^{2}}\left(I-2 \Delta_{S^{2} \times S^{2}}\right)^{\frac{1}{2}}(\mathcal{R} f)(g y, y) d y=\frac{1}{4 \pi}\left(\mathcal{R}^{*} \mathcal{R} f\right)(g), \quad g \in S O(3) .
$$

Thus, $\mathcal{R}^{-1}=\frac{1}{4 \pi} \mathcal{R}^{*}$.

One can verify that the following relations hold

$$
\mathcal{R} \mathcal{T}_{i j}^{k}(x, y)=\mathcal{T}_{i 1}^{k}(x) \overline{\mathcal{T}_{j 1}^{k}(y)}=\frac{4 \pi}{2 k+1} Y_{k}^{i}(x) \overline{Y_{k}^{j}(y)},
$$




$$
\begin{gathered}
\Delta_{S^{2} \times S^{2}} \mathcal{R} f=2 \mathcal{R} \Delta_{S O(3)} f, \quad f \in H_{2}(S O(3)), \\
\left(1-2 \Delta_{S^{2} \times S^{2}}\right)^{t / 2} \mathcal{R} f=\mathcal{R}\left(1-4 \Delta_{S O(3)}\right)^{t / 2} f, \quad f \in H_{t}(S O(3)), \quad t \geq 0, \\
\left(1-2 \Delta_{S^{2} \times S^{2}}\right)^{t / 2} g=\mathcal{R}\left(1-4 \Delta_{S O(3)}\right)^{t / 2} \mathcal{R}^{-1} g,
\end{gathered}
$$

where $g \in H_{t+1 / 2}^{\Delta}\left(S^{2} \times S^{2}\right), \quad t \geq 0$.

Theorem 2.6 (Reconstruction formula). Let

$$
G(x, y)=\mathcal{R} f(x, y)=\sum_{k=0}^{\infty} \sum_{i, j=1}^{2 k+1} \widehat{G}(k)_{i j} Y_{k}^{i}(x) \overline{Y_{k}^{j}(y)} \in H_{\frac{1}{2}+t}^{\Delta}\left(S^{2} \times S^{2}\right), t \geq 0,
$$

be a result of the Radon transform. Then the pre-image $f \in H_{t}(S O(3)), t \geq 0$, is given by

$$
\begin{aligned}
f & =\sum_{k=0}^{\infty} \sum_{i, j=1}^{2 k+1} \frac{(2 k+1)}{4 \pi} \widehat{G}(k)_{i j} \mathcal{T}_{i j}^{k}=\sum_{k=0}^{\infty} \sum_{i, j=1}^{2 k+1}(2 k+1) \widehat{f}(k)_{i j} \mathcal{T}_{i j}^{k} \\
& =\sum_{k=0}^{\infty}(2 k+1) \operatorname{trace}\left(\widehat{f}(k) \mathcal{T}^{k}\right) .
\end{aligned}
$$

\section{Generalized variational splines on compact Riemannian manifolds}

3.1. Generalized interpolating variational splines. For a given finite family of pairwise different submanifolds $\left\{M_{\nu}\right\}_{1}^{N}$ consider the following family of distributions

$$
F_{\nu}(f)=\int_{M_{\nu}} f
$$

which are well defined at least for functions in $H_{\varepsilon+d / 2}(M), \varepsilon>0$. In particular, if $M_{\nu}=x_{\nu} \in M$, then every $F_{\nu}$ is a Dirac measure $\delta_{x_{\nu}} \nu=1, \ldots, N, x_{\nu} \in M$.

Note that distributions $F_{\nu}$ belong to $H_{-\varepsilon-d / 2}(M)$ for any $\varepsilon>0$. Given a sequence of complex numbers $v=\left\{v_{\nu}\right\}, \nu=1,2, \ldots, N$, and a $t>d / 2$ we consider the following variational problem:

Find a function $u$ from the space $H_{t}(M)$ which has the following properties:

(1) $F_{\nu}(u)=v_{\nu}, \quad \nu=1,2, \ldots, N, \quad v=\left\{v_{\nu}\right\}$,

(2) $u$ minimizes functional $u \rightarrow\left\|(1-L)^{t / 2} u\right\|$.

We show that the solution to Variational problem exist and is unique for any $t>t_{0}$.

We need the following Independence Assumption in order to determine the Fourier coefficients of the solution.

Independence Assumption. There are functions $\vartheta_{\nu} \in C^{\infty}(M)$ such that

$$
F_{\nu}\left(\vartheta_{\mu}\right)=\delta_{\nu \mu},
$$

where $\delta_{\nu \mu}$ is the Kronecker delta.

Note, that this assumption implies in particular that the functionals $F_{\nu}$ are linearly independent. Indeed, if for certain coefficients $\gamma_{1}, \gamma_{2}, \ldots, \gamma_{N}$ we have a relation $\sum_{\nu=1}^{N} \gamma_{\nu} F_{\nu}=0$, then for any $1 \leq \mu \leq N$ we obtain that $0=\sum_{\nu=1}^{N} \gamma_{\nu} F_{\nu}\left(\vartheta_{\mu}\right)=\gamma_{\mu}$.

The families of distributions that satisfy our condition include in particular finite families of $\delta$ functionals and their derivatives. Another example is a set of integrals over submanifolds from a finite family of submanifolds of any codimension. 
The solution to the Variational Problem will be called a spline and will be denoted as $s_{t}(v)$. The set of all solutions for a fixed set of distributions $F=\left\{F_{\nu}\right\}$ and a fixed $t$ will be denoted as $S(F, t)$.

Definition 3.1. Given a function $f \in H_{t}(M)$ we will say that spline $s \in S(F, t)$ interpolates $f$ if

$$
F_{\nu}(f)=F_{\nu}(s) .
$$

Interpolating spline exists and unique (see below) and will be denoted as $s_{t}(f)$. Note, that from the point of view of the classical theory of variational splines it would be more natural to consider minimization of the functional $u \rightarrow\left\|L^{t / 2} u\right\|$. However, in the case of a general compact manifolds it is easer to work with the operator $I-L$ since this operator is invertible.

Our main result concerning variational splines is the following (see [49]).

Theorem 3.1. If $t>d$, then for any given sequence of scalars $v=\left\{v_{\nu}\right\}, \nu=$ $1,2, \ldots N$, the following statements are equivalent:

(1) $s_{t}(v)$ is the solution to the Variational Problem;

(2) $s_{t}(v)$ satisfies the the following equation in the sense of distributions

$$
(1-L)^{t} s_{t}(v)=\sum_{\nu=1}^{N} \alpha_{\nu} \overline{F_{\nu}}, \quad \alpha_{\nu}=\alpha_{\nu}\left(s_{t}(v)\right), \quad t>d
$$

where $\alpha_{1}, \ldots, \alpha_{N}$ form a solution of the $N \times N$ system

$$
\sum_{\nu=1}^{N} \beta_{\nu \mu} \alpha_{\nu}=v_{\mu}, \quad \alpha_{\nu}=\alpha_{\nu}\left(s_{t}(v)\right), \quad \mu=1, \ldots, N
$$

and

$$
\beta_{\nu \mu}=\sum_{j=0}^{\infty}\left(1+\lambda_{j}\right)^{-t} \overline{F_{\nu}\left(u_{j}\right)} F_{\mu}\left(u_{j}\right), \quad L u_{j}=-\lambda_{j} u_{j} ;
$$

(3) the Fourier series of $s_{t}(v)$ has the following form

$$
s_{t}(v)=\sum_{j=0}^{\infty} c_{j}\left(s_{t}(v)\right) u_{j}
$$

where

$$
c_{j}\left(s_{t}(v)\right)=\left\langle s_{t}(v), u_{j}\right\rangle=\left(1+\lambda_{j}\right)^{-t} \sum_{\nu=1}^{N} \alpha_{\nu}\left(s_{t}(v)\right) \overline{F_{\nu}\left(u_{j}\right)} .
$$

Remark 1. It is important to note that the system (3.4) is always solvable according to our uniqueness and existence result for the Variational Problem.

Remark 2. It is also necessary to note that the series (3.5) is absolutely convergent if $t>d$. Indeed, since functionals $F_{\nu}$ are continuous on the Sobolev space $H_{d / 2+\varepsilon}(M)$ we obtain that for any normalized eigen function $u_{j}$ which corresponds to the eigen value $\lambda_{j}$ the following inequality holds true

$$
\left|F_{\nu}\left(u_{j}\right)\right| \leq C(M, F)\left\|(1-L)^{d / 4} u_{j}\right\| \leq C(M, F)\left(1+\lambda_{j}\right)^{d / 4}, \quad F=\left\{F_{\nu}\right\}
$$

So

$$
\left|\overline{F_{\nu}\left(u_{j}\right)} F_{\mu}\left(u_{j}\right)\right| \leq C(M, F)\left(1+\lambda_{j}\right)^{d / 2}
$$


and

$$
\left|\left(1+\lambda_{j}\right)^{-t} \overline{F_{\nu}\left(u_{j}\right)} F_{\mu}\left(u_{j}\right)\right| \leq C(M, F)\left(1+\lambda_{j}\right)^{\left(t_{0}-t\right)} .
$$

It is known that the series

$$
\sum_{j} \lambda_{j}^{-\tau}
$$

which defines the $\zeta$-function of an elliptic second order operator, converges if $\tau>$ $d / 2$. This implies absolute convergence of (3.5) in the case $t>d$.

One can show that splines provide an optimal approximations to sufficiently smooth functions. Namely let $Q(F, f, t, K)$ be the set of all functions $h$ in $H_{t}(M)$ such that

(1) $F_{\nu}(h)=F_{\nu}(f), \nu=1,2, \ldots, N$,

(2) $\|h\|_{t} \leq K$, for a real $K \geq\left\|s_{t}(f)\right\|_{t}$.

The set $Q(F, f, t, K)$ is convex, bounded and closed. The following theorem (see [49]) shows that splines provide an optimal approximations to functions in $Q(F, f, t, K)$.

Theorem 3.2. The spline $s_{t}(f)$ is the symmetry center of $Q(F, f, t, K)$. This means that for any $h \in Q(F, f, t, K)$

$$
\left\|s_{t}(f)-h\right\|_{t} \leq \frac{1}{2} \operatorname{diam} Q(F, f, t, K) .
$$

3.2. Approximation by pointwise interpolation and approximation. To formulate our approximation theorem by variational splines in the case when the set of distributions $F_{i}$ is a set of delta functions we are using notion of a $\rho$-lattice which was introduced in Definition 2.3 and notion of bandlimited functions which was introduced in Definition 2.2.

Theorem 3.3. (Approximation Theorem [4], [4]) If $t>d / 2+k$ then there exist constants $C(M, t)>0, \rho(M)>0$ such that for any $0<\rho<\rho(M)$, any $\rho$-lattice $M_{\rho}$, any smooth function $f$ the following inequality holds true

$$
\left\|\left(s_{2^{m} d+t}(f)(x)-f(x)\right)\right\|_{C^{k}(M)} \leq\left(C(M, t) \rho^{2}\right)^{2^{m} d}\left\|(1-L)^{2^{m} d+t} f\right\|, \quad m=0,1, \ldots
$$

and if $f$ is $\omega$-bandlimited then

$$
\left\|\left(s_{2^{m} d+t}(f)(x)-f(x)\right)\right\|_{C^{k}(M)} \leq \omega^{t}\left(C(M, t) \rho^{2} \omega\right)^{2^{m} d}\|f\|,
$$

where $m=0,1, \ldots$

The first of these inequalities shows that convergence in $C^{k}(M)$ takes place when $\rho$ goes to zero and the index $2^{m} d+t$ is fixed.

The second inequality shows that right-hand side goes to zero for a fixed $\rho$ lattice $M_{\rho}$ as long as

$$
\rho<(C(M, t) \omega)^{-1 / 2}
$$

and $m$ goes to infinity. 
3.3. A sampling theorem and a cubature formula. Let $l_{x_{\nu}}^{k}$ denote a Lagrangian spline of order $k$, i.e. it takes value 1 at the node $x_{\nu}$ and zero at all other points of $M_{\rho}$. The last Theorem 3.3 can be formulated in the following form.

Theorem 3.4. (A Sampling Theorem) There exists a $c_{0}=c_{0}(M)$ such that for any $\omega>0$ and any $M_{\rho}=\left\{x_{\nu}\right\}$ with $\rho=c_{0} \omega^{-1 / 2}$ the following reconstruction formula holds in $L_{2}(M)$-norm

$$
f=\lim _{l \rightarrow \infty} \sum_{x_{\nu} \in M_{\rho}} f\left(x_{\nu}\right) l_{x_{\nu}}^{(k)}, k \geq d
$$

for all $f \in \mathbf{E}_{\omega}(L)$.

Note, that the right hand side of the last formula involves only values of $f$ on $M_{\rho}$. This statement is, in fact, a generalization of the classical Sampling Theorem to the case of compact Riemannian manifold.

The same result can be used to introduce a family of cubature formulas. To develop such formulas we introduce the notation

$$
\lambda_{\nu}^{(k)}=\int_{\mathbf{M}} l_{x_{\nu}}^{(k)}(x) d x,
$$

where $l_{x_{\nu}}^{k} \in S^{k}\left(M_{\rho}\right)$ is the Lagrangian spline at the node $x_{\nu}$.

The next theorem provides a cubature formula which is exact on variational splines.

Theorem 3.5. There exists a $c_{0}=c_{0}(M)$ such that the following statements hold true.

(1) For any $f \in H^{2 k}(M)$ one has

$$
\int_{\mathbf{M}} f d x \approx \sum_{x_{\nu} \in M_{\rho}} \lambda_{\nu}^{(k)} f\left(x_{\nu}\right), k \geq d,
$$

and the error given by the inequality

$$
\left|\int_{\mathbf{M}} f d x-\sum_{x_{\nu} \in M_{\rho}} \lambda_{\nu}^{(k)} f\left(x_{\nu}\right)\right| \leq\left(c_{0} \rho\right)^{k}\left\|L^{k / 2} f\right\|,
$$

for $k \geq d$. For a fixed function $f$ the right-hand side of (3.12) goes to zero as long as $\rho$ goes to zero.

(2) The formula (3.11) is exact for any variational spline $f \in S^{k}\left(M_{\rho}\right)$ of order $k$ with singularities on $M_{\rho}$.

By applying the Bernstein inequality we obtain the following theorem. This result explains our term "asymptotically correct cubature formulas".

Theorem 3.6. There exists a $c_{0}=c_{0}(M)$ such that for any $f \in \mathbf{E}_{\omega}(L)$ one has

$$
\left|\int_{\mathbf{M}} f d x-\sum_{x_{\nu} \in M_{\rho}} \lambda_{\nu}^{(k)} f\left(x_{\nu}\right)\right| \leq\left(c_{0} \rho \sqrt{\omega}\right)^{k}\|f\|,
$$

for $k \geq d$. If $c_{0} \rho \omega^{-1 / 2}<1$ the right-hand side in (3.13) goes to zero for all $f \in \mathbf{E}_{\omega}(L)$ as long as $k$ goes to infinity. 


\section{BANDLIMITED AND LOCALIZED PARSEVAL FRAMES ON HOMOGENEOUS MANIFOLDS}

In this section we assume that a manifold $M$ is homogeneous in the sense that it is of the form $M=G / H$, where $G$ is a compact Lie group and $H$ is its closed subgroup (see subsection 2.2). In this situation we consider spaces of bandlimited functions $\mathbf{E}_{\omega}(\mathcal{L}), \omega>0$, with respect to the Casimir operator $\mathcal{L}$ that was defined in (2.3). Our goal is to construct a tight bandlimited and localized frame in the space $L_{2}(M)$.

Let $g \in C^{\infty}\left(\mathbb{R}_{+}\right)$be a monotonic function such that supp $g \subset\left[0,2^{2}\right]$, and $g(s)=1$ for $s \in[0,1], 0 \leq g(s) \leq 1, s>0$. Setting $G(s)=g(s)-g\left(2^{2} s\right)$ implies that $0 \leq G(s) \leq 1, s \in \operatorname{supp} G \subset\left[2^{-2}, 2^{2}\right]$. Clearly, supp $G\left(2^{-2 j} s\right) \subset\left[2^{2 j-2}, 2^{2 j+2}\right], j \geq 1$. For the functions $\Phi(s)=\sqrt{g(s)}, \Phi\left(2^{-2 j} s\right)=\sqrt{G\left(2^{-2 j} s\right)}, j \geq 1$, one has

$$
\sum_{j \geq 0}\left(\Phi\left(2^{-2 j} s\right)\right)^{2}=1, s \geq 0 .
$$

Using the spectral theorem for $L$ one obtains

$$
\sum_{j \geq 0} \Phi^{2}\left(2^{-2 j} \mathcal{L}\right) f=f, \quad f \in L_{2}(M)
$$

and taking inner product with $f$ gives

$$
\|f\|^{2}=\sum_{j \geq 0}\left\langle\Phi^{2}\left(2^{-2 j} \mathcal{L}\right) f, f\right\rangle=\sum_{j \geq 0}\left\|\Phi\left(2^{-2 j} \mathcal{L}\right) f\right\|^{2} .
$$

Moreover, since the function $\Phi\left(2^{-2 j} s\right)$ has its support in $\left[2^{2 j-2}, 2^{2 j+2}\right]$ the elements $\Phi\left(2^{-2 j} \mathcal{L}\right) f$ are bandlimited to $\left[2^{2 j-2}, 2^{2 j+2}\right]$.

Expanding $f \in L_{2}(M)$ in terms of eigenfunctions of $\mathcal{L}$ we obtain

$$
\Phi\left(2^{-2 j} \mathcal{L}\right) f=\sum_{i} \Phi\left(2^{-2 j} \lambda_{i}\right) c_{i}(f) u_{i}, \quad c_{i}(f)=\left\langle f, u_{i}\right\rangle .
$$

Since for every $j$ function $\Phi\left(2^{-2 j} s\right)$ is supported in the interval $\left[2^{2 j-2}, 2^{2 j+2}\right]$ the function $\Phi\left(2^{-2 j} \mathcal{L}\right) f(x), x \in M$, is bandlimited and belongs to $\mathbf{E}_{2^{2 j+2}}(\mathcal{L})$. But then the function $\overline{\Phi\left(2^{-2 j} \mathcal{L}\right) f(x)}$ is also in $\mathbf{E}_{2^{2 j+2}}(\mathcal{L})$. Since

$$
\left|\Phi\left(2^{-2 j} \mathcal{L}\right) f(x)\right|^{2}=\left[\Phi\left(2^{-2 j} \mathcal{L}\right) f(x)\right]\left[\overline{\Phi\left(2^{-2 j} \mathcal{L}\right) f(x)}\right],
$$

one can use Theorem 2.2 to conclude that $\left|\Phi\left(2^{-2 j} \mathcal{L}\right) f\right|^{2} \in \mathbf{E}_{4 m 2^{2 j+2}}(\mathcal{L})$, where $m=\operatorname{dim} G, M=G / H$.

To summarize, we proved, that for every $f \in L_{2}(M)$ we have the following decomposition

$$
\sum_{j \geq 0}\left\|\Phi\left(2^{-2 j} \mathcal{L}\right) f\right\|^{2}=\|f\|^{2}, \quad\left|\Phi\left(2^{-2 j} \mathcal{L}\right) f(x)\right|^{2} \in \mathbf{E}_{4 m 2^{2 j+2}}(\mathcal{L}) .
$$

The next objective is to perform a discretization step. According to our Theorem 2.1 there exists a constant $c=c(M)>0$ such that for all integer $j$ if

$$
\rho_{j}=c\left(4 m 2^{2 j+2}+1\right)^{-1 / 2} \sim 2^{-j}, m=\operatorname{dim} G, M=G / H,
$$

then for any $\rho_{j}$-lattice $M_{\rho_{j}}$ one can find coefficients $b_{j, k}$ with

$$
b_{j, k} \sim \rho_{j}^{d}, \quad d=\operatorname{dim} M,
$$


for which the following exact cubature formula holds

$$
\left\|\Phi\left(2^{-2 j} \mathcal{L}\right) f\right\|^{2}=\sum_{k=1}^{J_{j}} b_{j, k}\left|\left[\Phi\left(2^{-2 j} \mathcal{L}\right) f\right]\left(x_{j, k}\right)\right|^{2},
$$

where $x_{j, k} \in M_{\rho_{j}},\left(k=1, \ldots, J_{j}=\operatorname{card}\left(M_{\rho_{j}}\right)\right)$.

For each $x_{j, k}$ we define the functions

$$
\begin{aligned}
\psi_{j, k}(y)=\overline{\mathcal{K}_{2-j}^{\Phi}}\left(x_{j, k}, y\right) & =\sum_{i} \bar{\Phi}\left(2^{-2 j} \lambda_{i}\right) \bar{u}_{i}\left(x_{j, k}\right) u_{i}(y), \\
\Psi_{j, k} & =\sqrt{b_{j, k}} \psi_{j, k} .
\end{aligned}
$$

We find that for all $f \in L_{2}(M)$,

$$
\|f\|^{2}=\sum_{j, k}\left|\left\langle f, \Psi_{j, k}\right\rangle\right|^{2} .
$$

Moreover, one can show [24], [51, 60] that the frame members $\psi_{j, k}$ are strongly localized on the manifold. All together it implies the following statement [24], [51.

Theorem 4.1. If $M$ is a homogeneous manifold, then the set of functions $\left\{\Psi_{j, k}\right\}$, constructed in (4.6)-(4.7) has the following properties:

(1) $\left\{\Psi_{j, k}\right\}$, is a Parseval frame in the space $L_{2}(M)$.

(2) Every function $\Psi_{j, k}$ is bandlimited to $\left[2^{2 j-2}, 2^{2 j+2}\right]$.

(3) For any $N>0$ there exists a $C(N)>0$ such that uniformly in $j$ and $k$

$$
\left|\psi_{j, k}(x)\right| \leq C(N) \frac{2^{d j}}{\max \left(1,2^{j} d\left(x, x_{j, k}\right)\right)^{N}}, \quad j \geq 0 .
$$

(4) The following reconstruction formula holds

$$
f=\sum_{j \geq 0}^{\infty} \sum_{k}\left\langle f, \Psi_{j, k}\right\rangle \Psi_{j, k}=\sum_{j \geq 0}^{\infty} \sum_{k} b_{j, k}\left\langle f, \psi_{j, k}\right\rangle \psi_{j, k}, \quad f \in L_{2}(M),
$$

with convergence in $L_{2}(M)$.

By using Theorems 2.1 and 2.2 one can easily obtain a following exact discrete formula for Fourier coefficients which uses only samples of $f$ on a sufficiently dense lattice.

Theorem 4.2. If $M$ is a homogeneous compact manifold then there exists a $c=$ $c(M)>0$ such that for any $\omega>0$, if $\rho_{\omega}=c(\omega+1)^{-1 / 2}$, then for any $\rho_{\omega}$-lattice $M_{\rho_{\omega}}=\left\{x_{\nu}\right\}_{\nu=1}^{N_{\omega}}$ of $M$, there exist positive weights $\mu_{\nu} \asymp(\omega+1)^{-d / 2}$, such that for every function $f$ in $\mathbf{E}_{\omega}(\mathcal{L})$ the Fourier coefficients $c_{i}(f)$

$$
c_{i}(f)=\int_{M} f \overline{u_{i}}, \quad-\mathcal{L} u_{i}=\lambda_{i} u_{i}, \quad \lambda_{i} \leq \omega,
$$

are given by the formulas

$$
c_{i}(f)=\sum_{\nu=1}^{N_{\omega}} \mu_{\nu} f\left(x_{\nu}\right) \overline{u_{i}}\left(x_{\nu}\right) .
$$

Theorems 4.1, 4.2, 2.1, and 2.2 can be used to prove another exact discrete formula for Fourier coefficients which uses frame functions 
Theorem 4.3. For a compact homogeneous manifold $M$ there exists a constant $c=c(M)>0$ such that for any natural $J$ if

$$
\rho_{J}=c 2^{-J}
$$

then for any $\rho_{J}$-lattice $M_{\rho_{J}}=\left\{x_{\nu}^{*}\right\}_{\nu=1}^{N_{\omega}}$, there exist positive weights

$$
\mu_{\nu}^{*} \asymp 2^{-d J}, \quad d=\operatorname{dim} M,
$$

such that the following formula holds

$$
f=\sum_{j=0}^{J} \sum_{k=1}^{n_{j}} \sum_{\nu=1}^{N_{J}} \mu_{\nu}^{*} f\left(x_{\nu}^{*}\right) \phi_{j, k}\left(x_{\nu}^{*}\right) \phi_{j, k}, \quad f \in \mathbf{E}_{\omega}(\mathcal{L}) .
$$

\section{Applications to Radon transform on $S^{d}$}

5.1. Approximate inversion of the spherical Radon transform using generalized splines. We consider approximate inversion of the Radon transform on $S^{d}$ (see subsection 2.4) when only a finite number of integrals over equatorial subspheres is given. Let $\left\{w_{\nu}\right\}, \nu=1,2, \ldots, N$, be a finite set of equatorial subspheres on $S^{d}$ of codimension one and distributions $F_{\nu}$ are given by formulas

$$
F_{\nu}(f)=\int_{w_{\nu}} f d x
$$

By solving corresponding variational problem we can find a spline $s_{t}(f) \in H_{t}\left(S^{d}\right)$ such that

$$
F_{\nu}\left(s_{t}(f)\right)=F_{\nu}(f), \nu=1,2, \ldots, N,
$$

and $s_{t}(f)$ minimizes norm $\left\|(1-\Delta)^{t / 2} s_{t}(f)\right\|$ where $\Delta$ is the Laplace-Beltrami operator in $L_{2}\left(S^{d}\right)$.

Our Theorem 3.1 in the case of the spherical Radon transform is summarized in the following statement.

Theorem 5.1. For a given symmetric $\rho$ - lattice $W=\left\{w_{\nu}\right\}$ of equatorial subspheres $\left\{w_{\nu}\right\}, \nu=1,2, \ldots, N$, an even smooth function $f$ and any $t>d / 2$ define $s_{t}(f)$ by the formula

$$
s_{t}(f)=\sum_{i, k} c_{i, k}\left(s_{t}(f)\right) Y_{k}^{i},
$$

where

$$
c_{i, k}\left(s_{t}(f)\right)=\left(1+\lambda_{i, k}\right)^{-t} \sum_{\nu=1}^{N} \alpha_{\nu}\left(s_{t}(f)\right) \int_{w_{\nu}} Y_{k}^{i} d x,
$$

and

$$
\begin{gathered}
\sum_{\nu=1}^{N} b_{\nu \mu} \alpha_{\nu}\left(s_{t}(f)\right)=v_{\mu}, \quad v_{\mu}=\int_{w_{\mu}} f d x, \quad \mu=1,2, \ldots, N, \\
b_{\nu \mu}=\sum_{i, k}\left(1+\lambda_{i, k}\right)^{-t} \int_{w_{\nu}} Y_{k}^{i} d x \int_{w_{\mu}} Y_{k}^{i} d x .
\end{gathered}
$$

The function $s_{t}(f)$ has the following properties.

(1) The function $s_{t}(f)$ is even. 
(2) Integrals of $s_{t}(f)$ over subspheres $w_{\nu}$ have prescribed values $v_{\nu}$

$$
\int_{w_{\nu}} s_{t}(f) d x=v_{\nu}, \nu=1,2, \ldots, N
$$

(3) among all functions that satisfy (2) function $s_{t}(f)$ minimizes the Sobolev norm

$$
\left\|(1-\Delta)^{t / 2} s_{t}(f)\right\|=\left(\sum_{\nu=1}^{N} \alpha_{\nu}\left(s_{t}(f)\right) v_{\nu}\right)^{1 / 2} .
$$

(4) The function $s_{t}(f)$ is the center of the convex set $Q(F, f, t, K)$ of all functions $h$ from $H_{t}\left(S^{d}\right)$ that satisfy both the condition (2) and the inequality

$$
\left\|(1-\Delta)^{t / 2} h\right\| \leq K,
$$

for any fixed $K \geq\left(\sum_{\nu=1}^{N} \alpha_{\nu}\left(s_{t}(f)\right) v_{\nu}\right)^{1 / 2}$. In other words for any $h \in$ $Q(F, f, t, K)$

$$
\left\|s_{t}(f)-h\right\|_{t} \leq \frac{1}{2} \operatorname{diam} Q(F, f, t, K)
$$

To prove item (1) one has to note that because of (2.7) integrals of $Y_{k}^{i}$ over great circles are zero as long as $k$ is odd. It implies that $c_{i, k}\left(s_{t}(f)\right)=0$ for every odd $k$ which means that decomposition of the corresponding spline $s_{t}(f)$ contains only harmonics $Y_{k}^{i}$ with even $k$.

5.2. A sampling theorem for the spherical Radon transform of bandlimited functions on $S^{d}$. According to Theorem 2.4 if $f \in H_{t}^{\text {even }}\left(S^{d}\right)$ then $R f \in$ $H_{t+(d-1) / 2}^{\text {even }}\left(S^{d}\right)$. Integral of $f$ over a great subsphere $w_{\nu}$ is the value of $R f$ at a point $x_{\nu}$ where $\left\{x_{\nu}\right\}=M_{\rho} \subset\left(S^{d}\right)$ is a $\rho$-lattice. For a fixed integer $m \geq 0$ and $t>d / 2$ we apply Theorem 3.1 to the manifold $S^{d}$ and the set of distributions $F=\left\{\delta_{x_{\nu}}\right\}$ where

$$
\delta_{x_{\nu}}(g)=g\left(x_{\nu}\right), \quad g \in C^{0}\left(S^{2} \times S^{2}\right), x_{\nu} \in M_{\rho} \subset S^{d}
$$

to construct spline $s_{\tau}(R f)$ with $\tau=2^{m} d+t+(d-1) / 2$ which interpolates $R f$ on $M_{\rho}=\left\{x_{\nu}\right\}$ and minimizes functional

$$
u \rightarrow\left\|(1-\Delta)^{\tau / 2} u\right\|
$$

where $\Delta$ is the Laplace-Beltrami operator in $L_{2}\left(S^{d}\right)$. Fourier coefficients of $s_{\tau}(R f)$ with respect to the basis $\left\{Y_{k}^{i}\right\}$ can be obtained by using Theorem 3.1. Let $\widehat{s}_{\tau}(R f)$ be the orthogonal projection (in the norm of $H_{t+(d-1) / 2}\left(S^{d}\right)$ ) of $s_{\tau}(R f)$ onto subspace $H_{\tau}^{\text {even }}\left(S^{d}\right)$. It means that $\widehat{s}_{\tau}(R f)$ has a representation of the form

$$
\widehat{s}_{\tau}(R f)(x)=\sum_{k} \sum_{i} c_{i}^{2 k}(R f) Y_{2 k}^{i}(x), \quad x \in S^{d},
$$

where

$$
c_{i}^{k}(R f)=c_{i}^{k}(R f ; m, t)=\int_{S^{d}} s_{\tau}(R f)(x) \overline{Y_{k}^{i}(x)} d x
$$

are Fourier coefficients of $s_{\tau}(R f)$. Applying (2.10) we obtain that the following function defined on $S^{d}$

$$
S_{\tau}(f)=R^{-1}\left(\widehat{s}_{\tau}(R f)\right)
$$


has a representation

$$
S_{\tau}(f)=\frac{\sqrt{\pi}}{\Gamma((d+1) / 2)} \sum_{k} \sum_{i} \frac{c_{2 k}^{i}(R f)}{r_{2 m}} Y_{2 k}^{i},
$$

where $r_{2 k}$ are defined in (2.9). Let us stress that these functions do not interpolate $f$ in any sense. However, as it will be shown they can be used to approximate $f$.

In the following theorem we assume that a $\rho$-lattice $M_{\rho}=\left\{x_{\nu}\right\}$ is a subset of points on the sphere $S^{d}$ and $M_{\rho}$ is dual to a collection of great subspheres $\left\{w_{\nu}\right\}$. we also assume that functions $S_{\tau}(f)$ constructed using vales of $R f$ on $M_{\rho}$. One can prove the following Theorem (see [49]).

Theorem 5.2. If $t>d / 2$ then there exists a constant $C=C(d, t)>0$ such that for any $\rho$-lattice $M_{\rho}=\left\{x_{\nu}\right\} \subset S^{d}$ with sufficiently small $\rho>0$ and any sufficiently smooth function $f$ on $S^{d}$ the following inequality holds true

$$
\left\|\left(S_{\tau}(f)-f\right)\right\|_{H_{t}\left(S^{d}\right)} \leq 2\left(C \rho^{2 d}\right)^{2^{m-1}}\|R f\|_{H_{\tau}\left(S^{d}\right)}, \quad \tau=2^{m} d+t+(d-1) / 2,
$$

for any $m=0,1, \ldots$. In particular, if a natural $k$ satisfies the inequality $t>k+d / 2$, then

$$
\left\|S_{\tau}(f)-f\right\|_{C^{k}\left(S^{d}\right)} \leq 2\left(C \rho^{2 d}\right)^{2^{m-1}}\|R f\|_{H_{\tau}\left(S^{d}\right)}
$$

for any $m=0,1, \ldots$

For an $\omega>0$ let us consider the subspace $\mathbf{E}_{\omega}^{\text {even }}\left(S^{d}\right)$ of even $\omega$-bandlimited functions on $S^{d}$. Clearly, this subspace is invariant under $R$.

Note, that for functions in $\mathbf{E}_{\omega}^{e v e n}\left(S^{d}\right)$ the following Bernstein-type inequality holds

$$
\left\|(1-\Delta)^{s} R f\right\|_{L^{2}\left(S^{d}\right)} \leq(1+\omega)^{s}\|R f\|_{L^{2}\left(S^{d}\right)} .
$$

As a consequence of the previous Theorem we obtain the next one (see 49.

Theorem 5.3. (Sampling Theorem For Radon Transform). If $t>d / 2$ then there exist constant $C=C(d, t)>0$ such that for any $\rho$-lattice $M_{\rho}=\left\{x_{\nu}\right\} \subset S^{d}$ with sufficiently small $\rho>0$ and any $f \in \mathbf{E}_{\omega}^{\text {even }}\left(S^{d}\right)$ one has the estimate

$$
\begin{gathered}
\left\|\left(S_{2^{m} d+t+(d-1) / 2}(f)-f\right)\right\|_{H_{t}\left(S^{d}\right)} \leq \\
2(1+\omega)^{t / 2+(d-1) / 2}\left(C \rho^{2}(1+\omega)\right)^{2^{m-1} d}\|R f\|_{L_{2}\left(S^{d}\right)},
\end{gathered}
$$

for any $m=0,1, \ldots$. In particular, if a natural $k$ satisfies the inequality $t>k+d / 2$, then

$$
\begin{gathered}
\left\|S_{2^{m} d+t+(d-1) / 2}(f)-f\right\|_{C^{k}\left(S^{d}\right)} \leq \\
2(1+\omega)^{t / 2+(d-1) / 2}\left(C \rho^{2}(1+\omega)\right)^{2^{m-1} d}\|R f\|_{L_{2}\left(S^{d}\right)},
\end{gathered}
$$

for any $m=0,1, \ldots$

This Theorem 5.3 shows that if $\rho<c \omega^{-1 / 2}$ then every $f \in \mathbf{E}_{\omega}\left(S^{d}\right)$ is completely determined by a finite set of values

$$
R f\left(x_{\nu}\right)=\int_{w_{\nu}} f, \quad x_{\nu} \in M_{\rho} .
$$

Moreover, it shows that $f$ can be reconstructed as a limit (when $m$ goes to infinity) of functions $S_{2^{m}} d+t+(d-1) / 2(f)$ which were constructed by using only the values of the Radon transform $R f\left(x_{\nu}\right)$. 
5.3. Exact formulas for Fourier coefficients of a bandlimited function $f$ on $S^{d}$ from a finite number of samples of $R f$. Theorem 4.2 can be used to obtain a discrete inversion formula for $R$.

Theorem 5.4. (Discrete Inversion Formula) There exists a $c=c(M)>0$ such that for any $\omega>0$, if $\rho_{\omega}=c \omega^{-1 / 2}$, then for any $\rho_{\omega}$-lattice $M_{\rho_{\omega}}=\left\{x_{\nu}\right\}_{\nu=1}^{m_{\omega}}$ of $S^{d}$, there exist positive weights $\mu_{\nu} \asymp \omega^{-d / 2}$, such that for every function $f$ in $\mathbf{E}_{\omega}\left(S^{d}\right)$ the Fourier coefficients $c_{k}^{i}(R f)$ of its Radon transform, i.e.

$$
R f(x)=\sum_{i, k} c_{k}^{i}(R f) \times Y_{k}^{i}(x), \quad k(k+1) \leq \omega, \quad x \in S^{d},
$$

are given by the formulas

$$
c_{k}^{i}(R f)=\sum_{\nu=1}^{m_{\omega}} \mu_{\nu} \times(R f)\left(x_{\nu}\right) \times Y_{k}^{i}\left(x_{\nu}\right) .
$$

The function $f$ can be reconstructed by means of the formula

$$
f=\frac{\sqrt{\pi}}{\Gamma((d+1) / 2)} \sum_{k} \sum_{i} \frac{c_{k}^{i}(R f)}{r_{k}} \times Y_{k}^{i}
$$

in which $k$ runs over all natural even numbers such that $k(k+1) \leq \omega$ and $r_{k}$ are defined in (2.9).

Proof. An application of Theorem 2.3 shows that if $k(k+1) \leq \omega$ then every product $Y_{k}^{i} \overline{Y_{k}^{j}}$, where $k(k+1) \leq \omega$ belongs to $\mathbf{E}_{2 \omega}\left(S^{d}\right)$.

By Theorem 2.1 there exists a positive constant $c=c(M)$, such that if $\rho_{\omega}=$ $c \omega^{-1 / 2}$, then for any $\rho_{\omega}$-lattice $M_{\rho_{\omega}}=\left\{x_{\nu}\right\}_{\nu=1}^{m_{\omega}}$ on $S^{d}$ there exist a set of positive weights $\mu_{\nu} \asymp \omega^{-d / 2}$ such that

$$
c_{k}^{i}(R f)=\int_{S^{d}}(R f)(x) \times \overline{Y_{k}^{i}(x)} d x=\sum_{\nu=1}^{m_{\omega}} \mu_{\nu} \times(R f)\left(x_{\nu}\right) \times \overline{Y_{k}^{i}\left(x_{\nu}\right)} .
$$

Thus,

$$
(R f)(x)=\sum_{k, i} c_{k}^{i}(R f) \times \overline{Y_{k}^{i}(x)} .
$$

Now the reconstruction formula of Theorem 2.6 implies the formula (5.9).

Without going to details we just mention that similar results can be obtained for the hemispherical Radon transform on $S^{d}$ (see [49]) and for the case of Radon transform of functions on domains see [53].

\section{Applications to the Radon transform on $S O(3)$}

6.1. Approximate inversion of the Radon transform on $S O(3)$ using generalized splines. Let $\left\{\left(x_{1}, y_{1}\right), \ldots,\left(x_{N}, y_{N}\right)\right\}$ be a set of pairs of points from $S O(3)$. In what follows we have to assume that our Independence Assumption (3.2) holds. It takes now the following form: there are smooth functions $\phi_{1}, \ldots, \phi_{N}$ on $S O(3)$ with

$$
\mathcal{R} \phi_{\mu}\left(x_{\nu}, y_{\nu}\right)=\delta_{\nu \mu}
$$


But it is obvious that for this condition to satisfy it is enough to assume that submanifolds $\mathcal{M}_{\nu}=x_{\nu} S O(2) y_{\nu}^{-1} \subset S O(3)$ are pairwise different (not necessarily disjoint).

Let $f$ be a function in $H_{t}(S O(3)), \quad t>\frac{1}{2} \operatorname{dim} S O(3)=3 / 2$ and

$$
v_{\nu}=\int_{\mathcal{M}_{\nu}} f, \quad \nu=1, \ldots, N, \quad v=\left\{v_{\nu}\right\} .
$$

According to Definition 3.1 we use notation $s_{t}(f)=s_{t}(v)$ for a function in $H_{t}(S O(3))$ such that for $\mathcal{M}_{\nu}=x_{\nu} S O(2) y_{\nu}^{-1}$ it satisfies (6.1) and minimizes the functional

$$
u \rightarrow\left\|\left(1-4 \Delta_{S O(3)}\right)^{t / 2} u\right\| .
$$

In this situation the results of section 3 can be summarized in the following statement which was proved in [5].

Theorem 6.1. Let $\left\{\left(x_{1}, y_{1}\right), \ldots,\left(x_{N}, y_{N}\right)\right\}$ be a subset of $S O(3) \times S O(3)$ such that submanifolds $\mathcal{M}_{\nu}=x_{\nu} S O(2) y_{\nu}^{-1} \subset S O(3), \nu=1, \ldots, N$, are pairwise different.

For a function $f$ in $H_{t}(S O(3)), t>3 / 2$, and a vector of measurements $v=\left(v_{\nu}\right)_{1}^{N}$ in (6.1) the solution of a constrained variational problem (6.1)-(6.2) is given by

$$
s_{t}(f)=\sum_{k=0}^{\infty} \sum_{i, j=1}^{2 k+1} c_{i j}^{k}\left(s_{t}(f)\right) \mathcal{T}_{i j}^{k}=\sum_{k=0}^{\infty} \operatorname{trace}\left(c_{k}\left(s_{t}(f)\right) \mathcal{T}^{k}\right),
$$

where $\mathcal{T}_{i j}^{k}$ are the Wigner polynomials. The Fourier coefficients $c_{k}\left(s_{t}(f)\right)$ of the solution are given by their matrix entries

$$
c_{i j}^{k}\left(s_{t}(f)\right)=\frac{4 \pi}{(2 k+1)(1+k(k+1))^{t}} \sum_{\nu=1}^{N} \alpha_{\nu}\left(s_{t}(f) Y_{k}^{i}\left(x_{\nu}\right) \overline{Y_{k}^{j}\left(y_{\nu}\right)},\right.
$$

where $\alpha\left(s_{t}(f)\right)=\left(\alpha_{\nu}\left(s_{t}(f)\right)\right)_{1}^{N} \in \mathbb{R}^{N}$ is the solution of

$$
\beta \alpha\left(s_{t}(f)\right)=f,
$$

with $\beta \in \mathbb{R}^{N \times N}$ given by

$$
\beta_{\nu \mu}=\sum_{k=0}^{\infty}(1+k(k+1))^{-t} C_{k}^{\frac{1}{2}}\left(x_{\nu} \cdot y_{\nu}\right) C_{k}^{\frac{1}{2}}\left(x_{\mu} \cdot y_{\mu}\right),
$$

where the Gegenbauer polynomials $C_{k}^{\frac{1}{2}}$ are given by the formulas

$$
\mathcal{C}_{k}^{\frac{1}{2}}(x \cdot y)=\frac{4 \pi}{2 k+1} \sum_{i=1}^{2 k+1} Y_{k}^{i}(x) \overline{Y_{k}^{i}(y)}
$$

for all $x, y \in S^{2}$ and $k=0,1,2 \ldots$. The function $s_{t}(f) \in H_{t}(S O(3))$ has the following properties:

(1) $s_{t}(f)$ has the prescribed set of measurements $v=\left(v_{\nu}\right)_{1}^{N}$ at points $\left(\left(x_{\nu}, y_{\nu}\right)\right)_{1}^{N}$;

(2) it minimizes the functional (6.2);

(3) the solution (6.3) is optimal in the sense that for every sufficiently large $K>0$ it is the symmetry center of the convex bounded closed set of all functions $h$ in $H_{t}(S O(3))$ with $\|h\|_{t} \leq K$ which have the same set of measurements $v=\left(v_{\nu}\right)_{1}^{N}$ at points $\left(\left(x_{\nu}, y_{\nu}\right)\right)_{1}^{N}$. 
6.2. A sampling theorem for Radon transform of bandlimited functions on $S O(3)$. According to Theorem 2.5 if $f \in H_{t}(S O(3))$ then $\mathcal{R} f \in H_{t+1 / 2}^{\Delta}\left(S^{2} \times S^{2}\right)$. Integral of $f$ over the manifold $x_{\nu} S O(2) y_{\nu}^{-1}$ is the value of $\mathcal{R} f$ at $\left(x_{\nu}, y_{\nu}\right)$ where $\left\{\left(x_{\nu}, y_{\nu}\right)\right\}=M_{\rho} \subset\left(S^{2} \times S^{2}\right)$ is a $\rho$-lattice. Note that dimension $d$ of the manifold $S^{2} \times S^{2}$ is four. For a fixed natural $m$ and $t>3 / 2$ we apply Theorem 3.1 to the manifold $S^{2} \times S^{2}$ and the set of distributions $F=\left\{\delta_{\nu}\right\}$ where

$$
\delta_{\nu}(g)=g\left(x_{\nu}, y_{\nu}\right), \quad g \in C^{0}\left(S^{2} \times S^{2}\right),\left(x_{\nu}, y_{\nu}\right) \in M_{\rho} \subset S^{2} \times S^{2},
$$

to construct spline $s_{2^{m}} d+(t+1)(\mathcal{R} f)$ which interpolates $\mathcal{R} f$ on $M_{\rho}=\left\{\left(x_{\nu}, y_{\nu}\right)\right\}$ and minimizes functional

$$
u \rightarrow\left\|\left(1-2 \Delta_{S^{2} \times S^{2}}\right)^{2^{m-1} d+(t+1) / 2} u\right\|, \quad d=4 .
$$

Fourier coefficients of $s_{2^{m}} d+(t+1)(\mathcal{R} f)$ with respect to the basis $\left\{Y_{k_{1}}^{i} Y_{k_{2}}^{j}\right\}$ can be obtained by using Theorem 3.1. Let $\widehat{s}_{2^{m}} d+(t+1)(\mathcal{R} f)$ be the orthogonal projection (in the norm of $\left.H_{t}\left(S^{2} \times S^{2}\right)\right)$ of $s_{2^{m}} d+(t+1)(\mathcal{R} f)$ onto subspace $H_{2^{m} d+(t+1)}^{\Delta}\left(S^{2} \times S^{2}\right)$. It means that $\widehat{s}_{2^{m}} d+(t+1)(\mathcal{R} f)$ has a representation of the form

$$
\widehat{s}_{2^{m} d+(t+1)}(\mathcal{R} f)(x, y)=\sum_{k} \sum_{i j} c_{i j}^{k}(\mathcal{R} f) Y_{k}^{i}(x) \overline{Y_{k}^{j}(y)}, \quad(x, y) \in S^{2} \times S^{2},
$$

where

$$
c_{i j}^{k}(\mathcal{R} f)=\int_{S^{2} \times S^{2}} s_{2^{m} d+(t+1)}(\mathcal{R} f)(x, y) \overline{Y_{k}^{i}(x)} Y_{k}^{j}(y) d x d y
$$

are Fourier coefficients of $s_{2^{m}} d+(t+1)(\mathcal{R} f)$. Applying (2.15) we obtain that the following function defined on $S O(3)$

$$
S_{2^{m}} d+(t+1)(f)(x)=\mathcal{R}^{-1}\left(\widehat{s}_{2^{m}} d+(t+1)(\mathcal{R} f)\right)(x), \quad d=4
$$

has a representation

$$
S_{2^{m} d+(t+1)}(f)(x)=\sum_{k} \sum_{i j} \frac{2 k+1}{4 \pi} c_{i j}^{k}(\mathcal{R} f) \mathcal{T}_{i j}^{k}(x), \quad d=4 .
$$

Let us stress that these functions do not interpolate $f$ in any sense. However, the following approximation results were proved in [5].

Theorem 6.2. If $t>3 / 2$ then there exist a constant $C=C(t)>0$ such that for any $\rho$-lattice $M_{\rho}=\left\{\left(x_{\nu}, y_{\nu}\right)\right\} \subset S^{2} \times S^{2}$ with sufficiently small $\rho>0$ and any sufficiently smooth function $f$ on $S O(3)$ the following inequality holds true

$$
\left\|\left(S_{\tau}(f)-f\right)\right\|_{H_{t}(S O(3))} \leq C_{1}(m) \rho^{2^{m+2}}\|\mathcal{R} f\|_{H_{\tau}\left(S^{2} \times S^{2}\right)}, .
$$

for $\tau=2^{m+2}+(t+1)$ and any $m=0,1, \ldots$. In particular, if a natural $k$ satisfies the inequality $t>k+3 / 2$, then

$$
\left\|S_{\tau}(f)-f\right\|_{C^{k}(S O(3))} \leq C_{1}(m) \rho^{2^{m+2}}\|\mathcal{R} f\|_{H_{\tau}\left(S^{2} \times S^{2}\right)}
$$

for any $m=0,1, \ldots$.

For an $\omega>0$ let us consider the space $\mathbf{E}_{\omega}(S O(3))$ of $\omega$-bandlimited functions on $S O(3)$ i.e. the span of all Wigner functions $T_{i j}^{k}$ with $k(k+1) \leq \omega$. As the formulas (2.12) and (2.15) show the Radon transform of such function is $\omega$-bandlimited on $S^{2} \times S^{2}$ in the sense its Fourier expansion involves only functions $Y_{k}^{i} \overline{Y_{k}^{j}}$ which are 
eigenfunctions of $\Delta_{S^{2} \times S^{2}}$ with eigenvalue $-2 k(k+1) \geq-2 \omega$. Let $\mathcal{E}_{\omega}\left(S^{2} \times S^{2}\right)$ be the span of $Y_{k}^{i}(\xi) \overline{Y_{k}^{j}(\eta)}$ with $k(k+1) \leq \omega$. Thus

$$
\mathcal{R}: \mathbf{E}_{\omega}(S O(3)) \rightarrow \mathcal{E}_{\omega}\left(S^{2} \times S^{2}\right) .
$$

For $f \in \mathbf{E}_{\omega}(S O(3))$ the following Bernstein-type inequality holds

$$
\left\|\left(1-2 \Delta_{S^{2} \times S^{2}}\right)^{\tau} \mathcal{R} f\right\|_{L^{2}\left(S^{2} \times S^{2}\right)} \leq(1+4 \omega)^{\tau}\|\mathcal{R} f\|_{L^{2}\left(S^{2} \times S^{2}\right)} .
$$

For the proof of the next theorem we refer to $[\underline{5}$.

Theorem 6.3. (Sampling Theorem For Radon Transform). If $t>3 / 2$ then there exist a constant $C=C(t)>0$ such that for any $\rho$-lattice $M_{\rho}=\left\{\left(x_{\nu}, y_{\nu}\right)\right\} \subset S^{2} \times S^{2}$ with sufficiently small $\rho>0$ and any $f \in \mathbf{E}_{\omega}(S O(3))$ one has the estimate

$$
\begin{gathered}
\left\|\left(S_{\tau}(f)-f\right)\right\|_{H_{t}(S O(3))} \leq \\
2(1+4 \omega)^{(t+1) / 2}\left[C \rho^{2}(1+4 \omega)\right]^{2^{m+1}}\|\mathcal{R} f\|_{L_{2}\left(S^{2} \times S^{2}\right)},
\end{gathered}
$$

for $\tau=2^{m+2}+(t+1)$ and any $m=0,1, \ldots$. In particular, if a natural $k$ satisfies the inequality $t>k+3 / 2$, then

$$
\left\|S_{\tau}(f)-f\right\|_{C^{k}(S O(3))} \leq 2(1+4 \omega)^{(t+1) / 2}\left[C \rho^{2}(1+4 \omega)\right]^{2^{m+1}}\|\mathcal{R} f\|_{L_{2}\left(S^{2} \times S^{2}\right)},
$$

for any $m=0,1, \ldots$.

This Theorem 6.3 shows that if $\rho<C(1+\omega)^{-1 / 2}$ then every $f \in \mathbf{E}_{\omega}(S O(3))$ is completely determined by a finite set of values

$$
\mathcal{R} f\left(x_{\nu}, y_{\nu}\right)=\int_{M_{\nu}} f
$$

where $M_{\nu}=x_{\nu} S O(2) y_{\nu}^{-1} \subset S O(3), \quad\left\{\left(x_{\nu}, y_{\nu}\right)\right\}=M_{\rho} \subset S^{2} \times S^{2}$. Moreover, it shows that $f$ can be reconstructed as a limit (when $m$ goes to infinity) of functions $S_{2^{m+2}+t}(f)$ which were constructed by using only the values of the Radon transform $\mathcal{R} f\left(x_{\nu}, y_{\nu}\right)$.

6.3. Exact formulas for Fourier coefficients of a bandlimited function $f$ on $S O(3)$ from a finite number of samples of $\mathcal{R} f$. Let $M_{\rho}=\left\{\left(x_{\nu}, y_{\nu}\right)\right\}$ be a metric $\rho$-lattice of $S^{2} \times S^{2}$. In what follows $\mathcal{E}_{\omega}\left(S^{2} \times S^{2}\right)$ will denote the span in the space $L^{2}\left(S^{2} \times S^{2}\right)$ of all $Y_{k}^{i} \overline{Y_{k}^{j}}$ with $k(k+1) \leq \omega$. Theorem 4.2 implies the following exam discrete reconstruction formula which uses only samples of $\mathcal{R} f$ on a sufficiently dense lattice.

Theorem 6.4. (Discrete Inversion Formula 6]) There exists a $c=c(M)>0$ such that for any $\omega>0$, if $\rho_{\omega}=c \omega^{-1 / 2}$, then for any $\rho_{\omega}$-lattice $M_{\rho_{\omega}}=\left\{\left(x_{\nu}, y_{\nu}\right)\right\}_{\nu=1}^{m_{\omega}}$ of $S^{2} \times S^{2}$, there exist positive weights $\mu_{\nu} \asymp \omega^{-2}$, such that for every function $f$ in $\mathbf{E}_{\omega}(S O(3))$ the Fourier coefficients $c_{i, j}^{k}(\mathcal{R} f)$ of its Radon transform, i.e.

$$
\mathcal{R} f(x, y)=\sum_{i, j, k} c_{i, j}^{k}(\mathcal{R} f) \times Y_{k}^{i}(x) \overline{Y_{k}^{j}}(y), \quad k(k+1) \leq \omega, \quad(x, y) \in S^{2} \times S^{2},
$$

are given by the formulas

$$
c_{i, j}^{k}(\mathcal{R} f)=\sum_{\nu=1}^{m_{\omega}} \mu_{\nu} \times(\mathcal{R} f)\left(x_{\nu}, y_{\nu}\right) \times Y_{k}^{i}\left(x_{\nu}\right) \overline{Y_{k}^{j}}\left(y_{\nu}\right) .
$$


The function $f$ can be reconstructed by means of the formula

$$
f(g)=\sum_{k} \sum_{i, j}^{2 k+1} \frac{(2 k+1)}{4 \pi} \times c_{i, j}^{k}(\mathcal{R} f) \times \mathcal{T}_{k}^{i, j}(g), \quad g \in S O(3),
$$

in which $k$ runs over all natural numbers such that $k(k+1) \leq \omega$.

\section{Conclusion}

During last few decades there was a strong demand for effective tools to perform interpolation, approximation, harmonic and multiscale analyses on such manifolds as the unit spheres $S^{2}$ and $S^{3}$ and the rotation group of $S O(3)$.

In our paper we develop a theory of variational interpolating splines on compact Riemannian manifolds. Our consideration includes in particular such problems as interpolation of a function by its values on a discrete set of points and interpolation by values of integrals over a family of submanifolds. The existence and uniqueness of interpolating variational spline on a Riemannian manifold is proven. Optimal properties of such splines are shown. The explicit formulas of variational splines in terms of the eigenfunctions of an appropriate elliptic differential operator are found. It is also shown that in the case of interpolation on discrete sets of points variational splines converge to a function in $C^{k}$ norms on manifolds. Variational splines are used to establish a generalization of the Classical Sampling Theorem and to develop cubature formulas on manifolds.

In a different venue we extend the well-developed field of time-frequency analysis based on frames and Shannon sampling to compact Riemannian manifolds by constructing bandlimited localized Parseval frames on compact manifolds which have many symmetries.

Applications of these results to the Radon transforms on the unit sphere $S^{d}$ and on the group of rotations $S O(3)$ are given.

The present study expands the important field of multiresolution analysis based on splines and frames from Euclidean spaces to compact Riemannian manifolds thus giving the means for modeling and analyzing various important complex phenomena.

\section{REFERENCES}

[1] P. Alfeld, M. Neamtu, L. L. Schumaker, Fitting scattered data on sphere-like surfaces using spherical splines, J. Comput. Appl. Math., 73, (1996), 5-43.

[2] Antoine JP, Vandergheynst P , Wavelets on the 2-sphere: a grouptheoretic approach, Appl Comput Harmon Anal 7(1999), 1-30.

[3] P.Baldi, G.Kerkyacharian, D.Marinucci, D.Picard, Asymptotics for Spherical Needlets, Annals of Statistics, Vol. 37, (2009), No. 3, 1150-1171.

[4] S. Bernstein, S. Ebert, Wavelets on $S 3$ and SO(3)their construction, relation to each other and Radon transform of wavelets on SO(3), Math. Methods Appl. Sci. 33 (2010), no. 16, 1895-1909.

[5] S. Bernstein, S. Ebert, I.Z. Pesenson, Generalized Splines for Radon Transform on Compact Lie Groups with Applications to Crystallography, J. Fourier Anal. Appl. 19 (2013), no. 1, 140-166.

[6] S. Bernstein, I.Z. Pesenson, The Radon transform on SO(3): motivations, generalizations, discretization, submitted; arXiv:1210.2652 2012.

[7] Bernstein, S., Schaeben, H., A one-dimensional Radon transform on SO(3) and its application to texture goniometry, Math. Meth. Appl. Sci., 28:1269-1289 (2005),

[8] Bernstein, S., Hielscher, R., Schaeben, H., The generalized totally geodesic Radon transform and its application to texture analysis, Math. Meth. Appl. Sci., 32:379-394 (2009), 
[9] K.G.van den Boogaart, R. Hielscher, J. Prestin and H. Schaeben, Kernel-based methods for inversion of the Radon transform on SO(3) and their applications to texture analysis, J. Comput. Appl. Math. 199 (2007),122-40,

[10] Dahlke S, Dahmen W, Schmitt W, Weinreich I, Multiresolution analysis and wavelets on $S^{2}$ and $S^{3}$, Numer Funct Anal Optim., 16(1995), 19-41.

[11] J.R. Driscoll and D.M. Healy, Computing Fourier Transforms and Convolutions on the 2Sphere, Adv. in Applied Math. 15(1994), 202-250.

[12] R. Duffin, A. Schaeffer, A class of nonharmonic Fourier series, Trans. AMS, 72, (1952), 341-366.

[13] N. Dyn, F.J. Narcovich, and J.D. Ward, Variational Principles and Sobolev-Type Estimates for generalized Interpolation on a Riemannian Manifold, Constr. Approx., 15(1999), 175-208.

[14] G. E. Fasshauer; L. L. Schumaker, Scattered data fitting on the sphere. Mathematical methods for curves and surfaces, II (Lillehammer, 1997), 117-166, Innov. Appl. Math., Vanderbilt Univ. Press, Nashville, TN, 1998.

[15] F. Filbir, H. Mhaskar, A quadrature formula for diffusion polynomials corresponding to a generalized heat kernel, J. Fourier Anal. Appl. 16 (2010), no. 5, 629-657.

[16] W. Freeden, An application of a summation formula to numerical computation of integrals over the sphere, Bull. Godsique 52 (1978), no. 3, 165-175.

[17] W. Freeden, On spherical spline interpolation and approximation, Math. Methods Appl. Sci. 3 (1981), no. 4, 551-575.

[18] W. Freeden, U. Windheuser, Spherical wavelet transform and its discretization, Adv. Comput. Math. 5, 51-94, (1996).

[19] W. Freeden, T. Gervens, M. Schreiner, Constructive approximation on the spheres. With applications to geomathematics, Numerical Mathematics and Scientific Computation, The Claredon Press, Oxford University Press, New York, 1998.

[20] W. Freeden, V. Michel, Multiscale Potential Theory (with Applications to Geoscience), Birkhuser, Boston, Basel, Berlin, (2004).

[21] W. Freeden, M. Schreiner, Biorthogonal locally supported wavelets on the sphere based on zonal kernel functions, J. Fourier Anal. Appl. 13 (2007), no. 6, 693-709.

[22] H. Führ, Painless Gabor expansions on homogeneous manifolds, Appl. Comput. Harmon. Anal. 26 (2009), no. 2, 200211.

[23] D. Geller and A. Mayeli, Nearly Tight Frames and Space-Frequency Analysis on Compact Manifolds (2009), Math. Z. 263 (2009), 235-264.

[24] D. Geller and I. Pesenson, Band-limited localized Parseval frames and Besov spaces on compact homogeneous manifolds, J. Geom. Anal. 21 (2011), no. 2, 334-371.

[25] D. Geller and I. Pesenson, Kolmogorov and Linear Widths of Balls in Sobolev and Besov Norms on Compact Manifolds, arXiv:1104.0632 1 [math.FA]

[26] D. Geller and D. Marinucci, Mixed needlets, J. Math. Anal. Appl. 375 (2011), no. 2, 610-630.

[27] K. Gröchenig, Foundations of time-frequency analysis, Birkhauser, 2001.

[28] T. Hangelbroek, ; F. J. Narcowich; X. Sun; J. D. Ward, Kernel approximation on manifolds II: the $L_{\infty}$ norm of the $L_{2}$ projector, SIAM J. Math. Anal. 43 (2011), no. 2, 662-684.

[29] Helgason, S., Differential Geometry and Symmetric Spaces, Academic, N.Y., 1962,

[30] S. Helgason, Geometric analysis on symmetric spaces, Second edition. Mathematical Surveys and Monographs, 39. American Mathematical Society, Providence, RI, 2008. xviii+637 pp. ISBN: 978-0-8218-4530-1

[31] K. Hesse, H.N. Mhaskar, I.H. Sloan, Quadrature in Besov spaces on the Euclidean sphere, J. Complexity 23 (2007), no. 4-6, 528-552.

[32] Hielscher, R., Potts, D., Prestin, J., Schaeben, H., Schmalz, M., The Radon transform on SO(3): a Fourier slice theorem and numerical inversion, Inverse Problems 24 (2008), no. 2, 025011, 21 pp.,

[33] Kakehi, T., Tsukamoto, C.,Characterization of images of Radon transform, Adv. Stud. Pure Math. (1993), 22, 101-16,

[34] Lai, Ming-Jun; Schumaker, Larry L. Spline functions on triangulations. Encyclopedia of Mathematics and its Applications, 110. Cambridge University Press, Cambridge, 2007. xvi+592 pp.

[35] Lyche T, Schumaker L , A multiresolution tensor spline method for fitting functions on the sphere, SIAM J Sci Comput, 22(2000), 724-746. 
[36] H.N. Mhaskar, F.J. Narcowich, J.D. Ward, Spherical Marcinkiewicz-Zygmund inequalities and positive quadrature, Math. Comp. 70 (2001), no. 235, 1113-1130.

[37] S. Mallat, Group invariant scattering, Comm. Pure Appl. Math. 65 (2012), no. 10, 1331-1398.

[38] D. Marinucci, G. Peccati, Random fields on the sphere. Representation, limit theorems and cosmological applications, London Mathematical Society Lecture Note Series, 389. Cambridge University Press, Cambridge, 2011. xii+341 pp. ISBN: 978-0-521-17561-6.

[39] D.Marinucci et al., Spherical Needlets for CMB Data Analysis, Monthly Notices of the Royal Astronomical Society, Vol. 383, (2008), pp. 539-545.

[40] F.J. Narcowich, J.D. Ward, Scattered data interpolation on spheres: Error estimates and locally supported basis functions, SIAM J. Math. Anal. 33 (2002) 1393-1410.

[41] F.J. Narcowich, P. Petrushev and J. Ward, Localized Tight frames on spheres, SIAM J. Math. Anal. 38, (2006), 574-594.

[42] I. Pesenson, Interpolation spaces on Lie groups, (Russian) Dokl. Akad. Nauk SSSR 246 (1979), no. 6, 1298-1303. (Engl. Transl. in Soviet Math. Dokl., v.20, No 3, pp. 611-616, 1979.)

[43] I. Pesenson, Nikolskii- Besov spaces connected with representations of Lie groups, (Russian) Dokl. Akad. Nauk SSSR 273 (1983), no. 1, 45-49.

[44] I. Pesenson, The Best Approximation in a Representation Space of a Lie Group, Dokl. Acad. Nauk USSR, v. 302, No 5, pp. 1055-1059, (1988) (Engl. Transl. in Soviet Math. Dokl., v.38, No 2, pp. 384-388, 1989.)

[45] I. Pesenson, The Bernstein Inequality in the Space of Representation of Lie group, Dokl. Acad. Nauk USSR 313 (1990), 86-90; English transl. in Soviet Math. Dokl. 42 (1991).

[46] I. Pesenson, A sampling theorem on homogeneous manifolds, Trans. Amer. Math. Soc. 352 (2000), no. 9, 4257-4269.

[47] I. Pesenson, An approach to spectral problems on Riemannian manifolds, Pacific J. of Math. Vol. 215(1), (2004), 183-199.

[48] I. Pesenson, Poincare-type inequalities and reconstruction of Paley-Wiener functions on manifolds, J. of Geometric Analysis , (4), 1, (2004), 101-121.

[49] I. Pesenson, Variational splines on Riemannian manifolds with applications to integral geometry, Adv. in Appl. Math. 33 (2004), no. 3, 548-572.

[50] I. Pesenson, Frames in Paley-Wiener spaces on Riemannian manifolds, in Integral Geometry and Tomography, Contemp. Math., 405, AMS, (2006), 137-153.

[51] I. Pesenson, D. Geller, Cubature formulas and discrete fourier transform on compact manifolds in "From Fourier Analysis and Number Theory to Radon Transforms and Geometry: In Memory of Leon Ehrenpreis" (Developments in Mathematics 28) by Hershel M. Farkas, Robert C. Gunning, Marvin I. Knopp and B. A. Taylor, Springer NY 2013.

[52] I. Pesenson, M. Pesenson, Approximation of Besov vectors by Paley-Wiener vectors in Hilbert spaces, Approximation Theory XIII: San Antonio 2010 (Springer Proceedings in Mathematics, Vol.13), by Marian Neamtu and Larry Schumaker, 249-263.

[53] I. Pesenson, $\varphi$-transform on domains in Euclidean spaces, submitted.

[54] I. Pesenson, Paley-Wiener-Schwartz nearly Parseval frames on noncompact symmetric spaces, will appear in a volume of Contemporary Mathematics.

[55] I. Pesenson, Multiresolution Analysis on Compact Riemannian Manifolds Multiscale Analysis and Nonlinear Dynamics: From Genes to the Brain (Annual Reviews of Nonlinear Dynamics and Complexity (VCH)) Misha Meyer Pesenson (Editor), Heinz Georg Schuster (Series Editor) WILEY-VCH 2013, pp. 65-83.

[56] G. Peyr, Manifold models for signals and images, Computer Vision and Image Understanding, 113 (2009) 249-260.

[57] E. Saucan; E. Appleboim; Y. Y. Zeevi, Image projection and representation on $S^{n}$. J. Fourier Anal. Appl. 13 (2007), no. 6, 711-727.

[58] I.J. Schoenberg, Positive definite functions on spheres, Duke. Math.J., 9(1942), 96-108.

[59] S. L. Sobolev, Cubature formulas on the sphere invariant under finite groups of rotations, Soviet Math. 3 (1962), 1307-1310.

[60] M. Taylor, Pseudodifferential Operators, Princeton University Press, 1981.

[61] G. Wahba, Spline interpolation and smoothing on the sphere, SIAM J Sci Stat Comput 2(1981), 5-16.

[62] G. Wahba, Surface fitting with scattered noisy data on Euclidean d-space and on the sphere, Rocky Mountain J. Math., 14(1984), 281-299. 
[63] G. Wahba, Spline models for observational data, In: CBMS-NSF regional conference series in applied mathematics, 59(1990). SIAM, Philadelphia 